\title{
Synthesis of macrolide-saccharide hybrids by ring closing metathesis of precursors derived from glycitols and benzoic acids
}

Marie-Christine Matos and Paul V. Murphy*

Centre for Synthesis and Chemical Biology, UCD School of Chemistry and Chemical Biology, UCD Conway Institute of Biomolecular and Biomedical Research, University College Dublin, Belfield, Dublin 4, Ireland.

Email: paul.v.murphy@ucd.ie

Index: $\quad$ Experimental Section and analytical data S-1-2 to S-1-29

$\mathrm{X}$-ray structures of $\mathbf{9 b}$ and $\mathbf{1 2} \quad \mathrm{S}-1-30$ 


\section{General Experimental Conditions}

Chemical shifts are reported relative to internal $\mathrm{Me}_{4} \mathrm{Si}$ in $\mathrm{CDCl}_{3}(\delta 0.0)$ or $\mathrm{HOD}$ for $\mathrm{D}_{2} \mathrm{O}(\delta$ 4.63) or $\mathrm{CD}_{2} \mathrm{HOD}(\delta 3.36)$ for ${ }^{1} \mathrm{H}$ and $\mathrm{CDCl}_{3}(\delta 77.0)$ or $\mathrm{CD}_{3} \mathrm{OD}(\delta 47.7)$ for ${ }^{13} \mathrm{C} .{ }^{1} \mathrm{H} \mathrm{NMR}$ signals were assigned with the aid of COSY. ${ }^{13} \mathrm{C}$ NMR signals were assigned with the aid of DEPT, HMBC and/or HSQC. Coupling constants are reported in hertz. The IR spectra were recorded using either thin film between $\mathrm{NaCl}$ plates or $\mathrm{KBr}$ discs, as specified. Thin layer chromatography (TLC) was performed on aluminium sheets precoated with silica gel and spots visualized by UV and charring with $\mathrm{H}_{2} \mathrm{SO}_{4}-\mathrm{EtOH}$ (1:20). Flash column chromatography was carried out with silica gel $60(0.040-0.630 \mathrm{~mm})$. Chromatography solvents were used as obtained from suppliers. Toluene, acetonitrile and $\mathrm{CH}_{2} \mathrm{Cl}_{2}$ were freshly distilled from calcium hydride. THF was freshly distilled from $\mathrm{Na} /$ Benzophenone. $\mathrm{MeOH}$ was distilled from $\mathrm{Mg}$.

\section{2-Bromobenzoic acid ethyl ester}

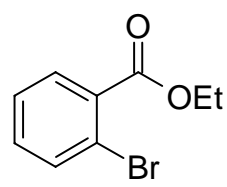

A solution of 2-bromobenzoic acid 13a (1 g, $4.97 \mathrm{mmol}, 1$ eq. $)$ and conc $\mathrm{H}_{2} \mathrm{SO}_{4}(0.65 \mathrm{~mL})$ in EtOH $(10 \mathrm{~mL})$ was heated at reflux for $6 \mathrm{~h}$ under $\mathrm{N}_{2}$. The solvent was removed under reduced pressure and water $(5 \mathrm{~mL})$ added to the residue which was then extracted with $\mathrm{Et}_{2} \mathrm{O}(3 \times 10$ $\mathrm{mL})$. The organic layer was washed with satd aq $\mathrm{NaHCO}_{3}(6 \mathrm{~mL})$, brine $(6 \mathrm{~mL})$ and dried $\left(\mathrm{MgSO}_{4}\right)$, filtered and the solvent removed under reduced pressure to give the title compound (1.01 g, 87\%, clear liquid which should be stored in a freezer); $\mathrm{R}_{\mathrm{f}} 0.70$ (7:3 cyclohexaneEtOAc); ${ }^{1} \mathrm{H}$ NMR $\left(300 \mathrm{MHz}, \mathrm{CDCl}_{3}\right): \delta 7.78(\mathrm{~m}, 1 \mathrm{H}), 7.66(\mathrm{~m}, 1 \mathrm{H}$, aromatic $\mathrm{H}), 7.35(\mathrm{~m}$, 2H), 4.41 (q, 2H, J 7.2 Hz), $1.42(\mathrm{q}, 3 \mathrm{H}, J 7.2 \mathrm{~Hz}) ;{ }^{13} \mathrm{C} \mathrm{NMR}\left(\mathrm{CDCl}_{3}\right): \delta 166.3(\mathrm{C}=\mathrm{O}), 134.3$ (d), 132.6 (s), 132.4 (d), 131.1 (d), 127.1 (d), 121.5 (s), 61.6 (t), 14.2 (q); IR (film, $\mathrm{CHCl}_{3}$ ): 
$\nu_{\max } 3068,2981,1729,1292,1251,1135,1108,1045,1029,746 \mathrm{~cm}^{-1}$. Anal. Calcd. for $\mathrm{C}_{9} \mathrm{H}_{9} \mathrm{O}_{2} \mathrm{Br}$ : C, 47.19 ; H, 3.96 ; Br, 34.88. Found: C, 47.04 ; H, 3.95 ; Br, 34.75.

\section{2-Vinylbenzoic acid ethyl ester}

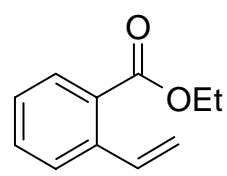

To a mixture of 2-bromobenzoic acid ethyl ester (5.42 g, $23.7 \mathrm{mmol})$ and $\mathrm{Pd}\left(\mathrm{PPh}_{3}\right)_{4}(547 \mathrm{mg}$, $0.47 \mathrm{mmol})$ in dry toluene $(60 \mathrm{~mL})$ was added vinyltributyltin $(8.30 \mathrm{~mL}, 28.4 \mathrm{mmol})$. The resulting mixture was heated for $24 \mathrm{~h}$ at $100{ }^{\circ} \mathrm{C}$ under $\mathrm{N}_{2}$. After filtration through celite, the solvent was removed in vacuo and the residue purified by chromatography [KF/Silica (1:9 w/w), $\mathrm{CH}_{2} \mathrm{Cl}_{2}$ ] to afford the title compound (3.62 $\mathrm{g}, 87 \%$, clear liquid); $\mathrm{R}_{\mathrm{f}} 0.50$ (9:1, cyclohexane-EtOAc); ${ }^{1} \mathrm{H}$ NMR $\left(300 \mathrm{MHz}, \mathrm{CDCl}_{3}\right): \delta 7.89(\mathrm{~d}, 1 \mathrm{H}, J 7.7 \mathrm{~Hz}), 7.59$ (d, 1H, $J$ $7.6 \mathrm{~Hz}), 7.47$ (dd \& t, 2H, $\left.J_{\mathrm{dd}} 17.6 \& 11.0 \mathrm{~Hz}, J_{\mathrm{t}} 7.6 \mathrm{~Hz}\right), 7.33(\mathrm{t}, 1 \mathrm{H}, J 7.7 \mathrm{~Hz}), 5.66(\mathrm{~d}, 1 \mathrm{H}, J$ $17.6 \mathrm{~Hz}), 5.36(\mathrm{~d}, 1 \mathrm{H}, J 11.0 \mathrm{~Hz}), 4.38(\mathrm{q}, 2 \mathrm{H}, J 7.1 \mathrm{~Hz}), 1.41(\mathrm{t}, 3 \mathrm{H}, J 7.1 \mathrm{~Hz}) ;{ }^{13} \mathrm{C} \mathrm{NMR}$ $\left(\mathrm{CDCl}_{3}\right): \delta 167.4(\mathrm{C}=\mathrm{O}), 139.4(\mathrm{~s}), 135.9$ (d), 131.9 (d), 130.2 (d), 129.0 (s), 127.3 (d), 127.1 (d), 116.3 (t), 61.0 (t), 14.3 (q); IR (film, $\mathrm{CHCl}_{3}$ ): $v_{\max }$ 3087, 3066, 2983, 1716, 1481, 1288, 1253, 1132, 1076, 917, 771, $717 \mathrm{~cm}^{-1}$. ES-HRMS: Found 177.0924, $\mathrm{C}_{11} \mathrm{H}_{13} \mathrm{O}_{2}$ requires $177.0916[\mathrm{M}+\mathrm{H}]^{+}$. 


\section{2-Vinylbenzoic acid 14a}<smiles>C=Cc1ccccc1C(=O)O</smiles>

To a solution of 2-vinylbenzoic acid, ethyl ester $(1.00 \mathrm{~g}, 5.68 \mathrm{mmol})$ in $\mathrm{MeOH}$-water $(2: 1 ; 60$ $\mathrm{mL})$ was added $\mathrm{LiOH} . \mathrm{H}_{2} \mathrm{O}(13.6 \mathrm{~g}, 323 \mathrm{mmol})$. The reaction mixture was stirred at $60{ }^{\circ} \mathrm{C}$ for $21 \mathrm{~h}$. The solution was then acidified with conc $\mathrm{HCl}$ until the $\mathrm{pH} \sim 0-1$. Water $(50 \mathrm{~mL})$ was added the mixture extracted with EtOAc $(3 \times 100 \mathrm{~mL})$. The combined organic layers were dried over $\mathrm{MgSO}_{4}$, filtered and the solvent was removed in vacuo. The residue was purified by chromatography (7:3, cyclohexane-EtOAc) to afford the title compound 14a $(801 \mathrm{mg}$, 95\%, white solid); $\mathrm{R}_{\mathrm{f}} 0.24$ (7:3, cyclohexane-EtOAc); mp $95.5{ }^{\circ} \mathrm{C}$ (water); ${ }^{1} \mathrm{H}$ NMR (300 $\left.\mathrm{MHz}, \mathrm{CDCl}_{3}\right): \delta 8.04(\mathrm{dd}, 1 \mathrm{H}, J 7.9 \& 1.0 \mathrm{~Hz}), 7.58(\mathrm{~m}, 3 \mathrm{H}), 7.38(\mathrm{td}, 1 \mathrm{H}, J 7.5 \& 1.3 \mathrm{~Hz}$ $5.68(\mathrm{dd}, 1 \mathrm{H}, J 17.4 \& 1.2 \mathrm{~Hz}), 5.39(\mathrm{dd}, 1 \mathrm{H}, J 11.0 \& 1.2 \mathrm{~Hz}) ;{ }^{13} \mathrm{C}$ NMR $(400 \mathrm{MHz}$, $\left.\mathrm{CDCl}_{3}\right): \delta 172.4(\mathrm{C}=\mathrm{O}), 140.6(\mathrm{~d}), 136.0$ (d), 133.1 (d), 131.3 (d), 127.6 (s), 127.5 (d), 127.1 (s), 116.8 (t); IR (film, MeOH) $v_{\max }$ 1683, 1303, 1268, 906, $767 \mathrm{~cm}^{-1}$. ES-HRMS: Found 147.0444, $\mathrm{C}_{9} \mathrm{H}_{7} \mathrm{O}_{2}$ requires $147.0446[\mathrm{M}-\mathrm{H}]^{-}$

\section{2-Bromo-5-methoxybenzoic acid, ethyl ester}<smiles>CCOC(=O)c1cc(OC)ccc1Br</smiles>

A solution of 2-bromo-5-methoxybenzoic acid $\mathbf{1 3 b}(1.01 \mathrm{~g}, 4.38 \mathrm{mmol})$ in EtOH $(20 \mathrm{~mL})$ with conc $\mathrm{H}_{2} \mathrm{SO}_{4}(0.73 \mathrm{~mL})$ was heated at reflux for $30 \mathrm{~h}$. The reaction was cooled, quenched with a satd soln of $\mathrm{NaHCO}_{3}(20 \mathrm{~mL})$, extracted with $\mathrm{Et}_{2} \mathrm{O}(3 \times 50 \mathrm{~mL})$, washed with brine (30 $\mathrm{mL}$ ), dried over $\mathrm{Na}_{2} \mathrm{SO}_{4}$, filtered and the solvent was removed in vacuo. The residue was purified by chromatography $(1: 1$, cyclohexane-EtOAc) to afford the title compound $(1.01 \mathrm{~g}$, 
$90 \%$, clear liquid); $\mathrm{R}_{\mathrm{f}} 0.57$ (1:1, cyclohexane-EtOAc); ${ }^{1} \mathrm{H}$ NMR $\left(300 \mathrm{MHz}, \mathrm{CDCl}_{3}\right): \delta 7.52(\mathrm{~d}$, $1 \mathrm{H}, J 8.8 \mathrm{~Hz}), 7.29(\mathrm{~d}, 1 \mathrm{H}, J 3.0 \mathrm{~Hz}), 6.87(\mathrm{dd}, 1 \mathrm{H}, J 8.8 \& 3.0 \mathrm{~Hz}), 3.40(\mathrm{q}, 2 \mathrm{H}, J 7.1 \mathrm{~Hz})$, $3.81(\mathrm{~s}, 3 \mathrm{H}), 1.40(\mathrm{t}, 3 \mathrm{H}, \mathrm{J} 7.1 \mathrm{~Hz}) ;{ }^{13} \mathrm{C} \mathrm{NMR}\left(\mathrm{CDCl}_{3}\right): \delta 166.1(\mathrm{C}=\mathrm{O}), 158.5(\mathrm{~s}), 134.9(\mathrm{~d})$, 133.22 (s), 133.18 (s), 118.7 (d), 116.2 (d), 111.8 (d), 61.7 (t), 55.6 (q), 14.2 (q) ; IR (film, $\left.\mathrm{CHCl}_{3}\right): v_{\max } 2981,2937,2906,2838,1729,1592,1569,1471,1290,1249,1226,1105,1049$, 1018, 813, $777 \mathrm{~cm}^{-1}$. ES-HRMS: Found 258.9962, $\mathrm{C}_{10} \mathrm{H}_{12} \mathrm{O}_{3} \mathrm{Br}$ requires $258.9970[\mathrm{M}+\mathrm{H}]^{+}$.

\section{5-Methoxy-2-vinylbenzoic acid, ethyl ester}

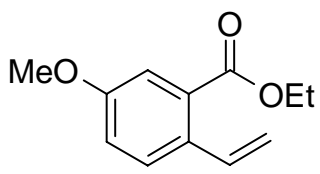

To a mixture of 2-bromo-5-methoxybenzoic acid, ethyl ester (458 mg, $1.77 \mathrm{mmol}$ ) and $\operatorname{Pd}\left(\mathrm{PPh}_{3}\right)_{4}(102 \mathrm{mg}, 0.09 \mathrm{mmol})$ in dry toluene $(15 \mathrm{~mL})$ was added vinyltributyltin $(0.57 \mathrm{~mL}$, $1.95 \mathrm{mmol}$ ). The mixture was heated for $18 \mathrm{~h}$ at $100{ }^{\circ} \mathrm{C}$ under $\mathrm{N}_{2}$. After filtration through celite, the solvent was removed in vacuo and the residue purified by chromatography [KF/Silica (1:9,w/w), $\mathrm{CH}_{2} \mathrm{Cl}_{2}$ ) to afford the title compound (326 mg, $89 \%$, oil); $\mathrm{R}_{\mathrm{f}} 0.28$ (9:1,

cyclohexane-EtOAc); ${ }^{1} \mathrm{H}$ NMR (300 MHz, $\left.\mathrm{CDCl}_{3}\right): \delta 7.52(\mathrm{~d}, 1 \mathrm{H}, J 8.7 \mathrm{~Hz}), 7.37(\mathrm{~m}, 2 \mathrm{H})$, $7.02(\mathrm{dd}, 1 \mathrm{H}, J 8.7 \& 2.6 \mathrm{~Hz}), 5.55(\mathrm{~d}, 1 \mathrm{H}, J 17.4 \mathrm{~Hz}), 5.25(\mathrm{~d}, 1 \mathrm{H}, J 11.0 \mathrm{~Hz}), 4.37$ (q, 2H, J $7.1 \mathrm{~Hz}), 3.84\left(\mathrm{~s}, 3 \mathrm{H}, 1.39(\mathrm{t}, 3 \mathrm{H}, J 7.1 \mathrm{~Hz}) ;{ }^{13} \mathrm{C} \mathrm{NMR}\left(\mathrm{CDCl}_{3}\right): \delta 167.3(\mathrm{C}=\mathrm{O}), 158.5(\mathrm{~s})\right.$, $135.2(\mathrm{~d}), 132.0(\mathrm{~s}), 130.0(\mathrm{~s}), 128.3(\mathrm{~d}), 118.2(\mathrm{~d}), 114.6(\mathrm{t}$ and d), $61.1(\mathrm{t}), 55.4(\mathrm{q}), 14.3(\mathrm{q})$; IR (film, $\mathrm{CHCl}_{3}$ ): $v_{\max }$ 3087, 2979, 2940, 2906, 2838, 1720, 1606, 1565, 1494, 1463, 1365, 1319, 1288, 1255, 1226, 1182, 1070, 1024, 829, $790 \mathrm{~cm}^{-1}$. ES-HRMS: Found 207.1011, $\mathrm{C}_{12} \mathrm{H}_{15} \mathrm{O}_{3}$ requires $207.1021[\mathrm{M}+\mathrm{H}]^{+}$. 


\section{5-Methoxy-2-vinylbenzoic acid 14b}<smiles>C=Cc1ccc(OC)cc1C(=O)O</smiles>

To a solution of 5-methoxy-2-vinylbenzoic acid, ethyl ester (300 mg, $1.46 \mathrm{mmol})$ in a mixture of $\mathrm{MeOH}$ and water $(2: 1 ; 20 \mathrm{~mL})$ was added $\mathrm{LiOH}_{2} \mathrm{H}_{2} \mathrm{O}(3.5 \mathrm{~g}, 83 \mathrm{mmol})$. The reaction mixture stirred at $60^{\circ} \mathrm{C}$ for $17 \mathrm{~h}$. The solution was then acidified with conc $\mathrm{HCl}$ to $\mathrm{pH} \sim 1$. Water $(10 \mathrm{~mL})$ was added and the compound extracted with EtOAc $(3 \times 20 \mathrm{~mL})$. The combined organic layers were dried over $\mathrm{MgSO}_{4}$, filtered and the solvent removed in vacuo. The residue was purified by chromatography $(1: 1$, cyclohexane-EtOAc) to afford $\mathbf{1 4 b}$ (189 mg, $73 \%$, white solid); $\mathrm{R}_{\mathrm{f}} 0.41$ (1:1, cyclohexane-EtOAc); mp $103.4{ }^{\circ} \mathrm{C}$ (water); ${ }^{1} \mathrm{H}$ NMR $\left(300 \mathrm{MHz}, \mathrm{CDCl}_{3}\right): \delta 10.75($ br s, $1 \mathrm{H}), 7.52(\mathrm{~m}, 3 \mathrm{H}), 7.10(\mathrm{dd}, 1 \mathrm{H}, J 8.7 \& 2.5 \mathrm{~Hz}), 5.59(\mathrm{~d}$, $1 \mathrm{H}, J 17.4 \mathrm{~Hz}), 5.29(\mathrm{~d}, 1 \mathrm{H}, J 11.0 \mathrm{~Hz}) ;{ }^{13} \mathrm{C} \mathrm{NMR}\left(\mathrm{CDCl}_{3}\right): \delta 172.9(\mathrm{C}=\mathrm{O}), 158.8(\mathrm{~s}), 135.4$ (d), 133.3 (s), 128.8 (d), 128.0 (s), 119.9 (d), 115.2 (t), 115.1 (d), 55.5 (q) ; IR (film, MeOH) : $v_{\max } 3421,1641,1496,1419,1324,1270,1240 \mathrm{~cm}^{-1}$. ES-HRMS: Found 177.0550, $\mathrm{C}_{10} \mathrm{H}_{9} \mathrm{O}_{3}$ requires $177.0552[\mathrm{M}-\mathrm{H}]^{-}$. Anal. Calcd. for $\mathrm{C}_{10} \mathrm{H}_{10} \mathrm{O}_{3}$ : C, 67.41; H, 5.66. Found: $\mathrm{C}, 67.14$; H, 5.65.

\section{2-Hydroxy-4-methoxybenzoic acid, methyl ester}

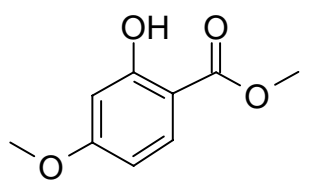

To an ice-cooled solution of 4-methoxysalicylic acid 15 (500 mg, $2.98 \mathrm{mmol})$ in THF (15 $\mathrm{mL})$ was added dropwise DBU $(0.45 \mathrm{~mL}, 2.98 \mathrm{mmol})$ over 5 min. Iodomethane $(0.19 \mathrm{~mL}$, $2.98 \mathrm{mmol}$ ) was then added and the mixture stirred at room temperature for $40 \mathrm{~h}$. The precipitate was filtered off and the solvent was removed in vacuo and the residue purified by 
chromatography $(1: 1$, cyclohexane-EtOAc) to afford the title compound (415 $\mathrm{mg}, 77 \%$, white crystalline solid); $\mathrm{R}_{\mathrm{f}} 0.83$ (1:1, cyclohexane-EtOAc); mp $44.3{ }^{\circ} \mathrm{C}$ (EtOAc); ${ }^{1} \mathrm{H}$ NMR (300 $\left.\mathrm{MHz}, \mathrm{CDCl}_{3}\right): \delta 10.97(\mathrm{~d}, 1 \mathrm{H}, J 0.7 \mathrm{~Hz}), 7.72(\mathrm{~d}, 1 \mathrm{H}, J 9.2 \mathrm{~Hz}), 6.42(\mathrm{~m}, 2 \mathrm{H}), 3.91(\mathrm{~s}, 3 \mathrm{H})$, $3.81(\mathrm{~s}, 3 \mathrm{H}) ;{ }^{13} \mathrm{C} \mathrm{NMR}\left(\mathrm{CDCl}_{3}\right): \delta 170.4(\mathrm{C}=\mathrm{O}), 165.6(\mathrm{~s}), 163.7(\mathrm{~s}), 131.2(\mathrm{~d}), 107.5(\mathrm{~d})$, 105.4 (s), 100.6 (d), 55.4 (q), 51.9 (q) ; IR (film, $\mathrm{CHCl}_{3}$ ): $v_{\max } 2962,2917,2850,1673,1623$, $1438,1353,1263,1228,1143,1024,836,775 \mathrm{~cm}^{-1}$. ES-HRMS: Found 181.0492, $\mathrm{C}_{9} \mathrm{H}_{9} \mathrm{O}_{4}$ requires $181.0501[\mathrm{M}-\mathrm{H}]^{-}$.

\section{4-Methoxy-2-trifluoromethanesulfonyloxy-benzoic acid, methyl ester}

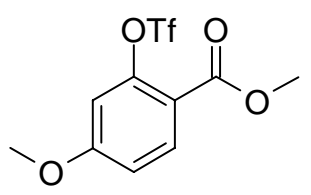

To a solution of 2-hydroxy-4-methoxybenzoic acid, methyl ester (100 mg, $0.55 \mathrm{mmol})$ in dry pyridine $(2 \mathrm{~mL})$ was added triflic anhydride $(0.11 \mathrm{~mL}, 0.64 \mathrm{mmol})$ dropwise over $5 \mathrm{~min}$ under $\mathrm{N}_{2}$. The reaction mixture was then stirred at room temperature for $21 \mathrm{~h}$ and then quenched with water $(6 \mathrm{~mL})$ and a solution of $\mathrm{HCl}(2 \mathrm{M}, 6 \mathrm{~mL})$ was later added. The mixture was acidified to $\mathrm{pH} \sim 1$ with conc $\mathrm{HCl}$ and extracted with EtOAc $(3 \times 25 \mathrm{~mL})$. The combined organic layers were washed with satd aq $\mathrm{NaHCO}_{3}(10 \mathrm{~mL})$ and dried over $\mathrm{MgSO}_{4}$. After filtration, the solvent was removed in vacuo and the residue purified by chromatography $(9: 1$, cyclohexane-EtOAc) to afford the title compound ( $155 \mathrm{mg}, 90 \%$, white solid) ; $\mathrm{R}_{\mathrm{f}} 0.45$ (9:1, cyclohexane-EtOAc) ; mp $41.7{ }^{\circ} \mathrm{C} ;{ }^{1} \mathrm{H}$ NMR $\left(300 \mathrm{MHz}, \mathrm{CDCl}_{3}\right): \delta 8.05$ (dd, $1 \mathrm{H}, J 8.8 \& 1.0$ Hz), 6.95 (ddd, 1H, J 8.9, 2.5 \& $1.2 \mathrm{~Hz}), 6.77$ (d, 1H, J $2.1 \mathrm{~Hz}), 3.92$ (s, 3H), 3.87 (s, 3H) ; ${ }^{13} \mathrm{C}$ NMR $\left(\mathrm{CDCl}_{3}\right): \delta 163.9(\mathrm{C}=\mathrm{O}), 149.6\left(\mathrm{CF}_{3}\right), 134.1(\mathrm{~d}), 120.8(\mathrm{~s}), 116.6(\mathrm{~s}), 116.2(\mathrm{~s})$, 113.4 (d), 108.8 (d), 55.9 (q), 52.2 (q); ${ }^{19} \mathrm{~F}$ NMR $\left(\mathrm{CDCl}_{3}\right): \delta$-85.8, -86.5, -87.1; IR (film, $\left.\mathrm{CHCl}_{3}\right): v_{\max } 3008,2958,2908,2846,2605,2360,1725,1618,1571,1508,1429,1276,1207$, 1139, 1072, 1029, 948, 831, 771, 690, $653 \mathrm{~cm}^{-1}$. ES-HRMS: Found 315.0148, $\mathrm{C}_{10} \mathrm{H}_{10} \mathrm{O}_{6} \mathrm{~F}_{3} \mathrm{~S}$ 
requires $315.0150[\mathrm{M}+\mathrm{H}]^{+}$. Anal. Calcd. for $\mathrm{C}_{10} \mathrm{H}_{9} \mathrm{~F}_{3} \mathrm{O}_{6} \mathrm{~S}: \mathrm{C}, 38.22 ; \mathrm{H}, 2.89 ; \mathrm{F}, 18.14 ; \mathrm{S}$, 10.20. Found: C, 38.12 ; H, 2.80 ; F, 18.21 ; S, 10.43.

\section{4-Methoxy-2-vinylbenzoic acid, methyl ester}

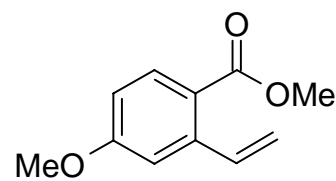

To a solution of 4-methoxy-2-trifluoromethanesulfonyloxy-benzoic acid, methyl ester (2.83 g, $9.00 \mathrm{mmol}), \mathrm{LiCl}(1.19 \mathrm{~g}, 28.2 \mathrm{mmol}, 3.1$ eq. $)$ and $\mathrm{Pd}\left(\mathrm{PPh}_{3}\right)_{4}(312 \mathrm{mg}, 0.27 \mathrm{mmol})$ in dry THF ( $88 \mathrm{~mL})$ was added vinyltributytin $(2.75 \mathrm{~mL}, 9.27 \mathrm{mmol}, 1.03$ eq.) at room temperature under $\mathrm{N}_{2}$. The mixture was then heated at reflux for $8 \mathrm{~h}$. After filtration through silica, the solvent was removed in vacuo to give a residue which was purified by chromatography [KFSilica (1:9, w/w), $\left.\mathrm{CH}_{2} \mathrm{Cl}_{2}\right]$ to give the title compound (463 mg, $27 \%$, oil) ; $\mathrm{R}_{\mathrm{f}} 0.36$ (4:1, cyclohexane-EtOAc) ; ${ }^{1} \mathrm{H}$ NMR $\left(300 \mathrm{MHz}, \mathrm{CDCl}_{3}\right): \delta 7.91(\mathrm{~d}, 1 \mathrm{H}, J 8.8 \mathrm{~Hz}), 7.55(\mathrm{dd}, 1 \mathrm{H}, J$ $17.4 \& 10.9 \mathrm{~Hz}), 7.04(\mathrm{~d}, 1 \mathrm{H}, J 1.9 \mathrm{~Hz}), 6.83(\mathrm{dd}, 1 \mathrm{H}, J 8.8 \& 2.0 \mathrm{~Hz}), 5.62(\mathrm{~d}, 1 \mathrm{H}, J 17.4$ $\mathrm{Hz}), 5.35(\mathrm{~d}, 1 \mathrm{H}, J 10.9 \mathrm{~Hz}), 3.87(\mathrm{~s}, 6 \mathrm{H}) ;{ }^{13} \mathrm{C} \mathrm{NMR}\left(\mathrm{CDCl}_{3}\right): \delta 167.3(\mathrm{C}=\mathrm{O}), 162.5(\mathrm{~s})$, $142.4(\mathrm{~s}), 136.4(\mathrm{~d}), 132.7(\mathrm{~d}), 120.8(\mathrm{~s}), 116.4(\mathrm{t}), 112.8(\mathrm{~d}), 112.4(\mathrm{~d}), 55.4(\mathrm{q}), 51.8$ (q) ; IR (film, $\mathrm{CHCl}_{3}$ ) : $v_{\max } 3085,2960,2913,2838,1712,1600,1563,1490,1432,1321,1261$, 1238, 1189, 1128, 1091, 1056, 1025, 917, 875, 862, 809, $784 \mathrm{~cm}^{-1}$. ES-HRMS: Found 193.0856, $\mathrm{C}_{11} \mathrm{H}_{13} \mathrm{O}_{3}$ requires $193.0865[\mathrm{M}+\mathrm{H}]^{+}$. Starting material was also recovered unreacted $(1.49 \mathrm{~g}, 52 \%)$. 


\section{4-Methoxy-2-vinylbenzoic acid 16}<smiles>C=Cc1cc(OC)ccc1C(=O)O</smiles>

4-Methoxy-2-vinylbenzoic acid, methyl ester (112 $\mathrm{mg}, 0.58 \mathrm{mmol})$ was treated with LiOH. $\mathrm{H}_{2} \mathrm{O}$ (1.4 g, $\left.33.2 \mathrm{mmol}\right)$ as described above and gave 16 (53 mg, 51\%, white solid); $\mathrm{R}_{\mathrm{f}} 0.44$ (1:1, cyclohexane-EtOAc); mp $149.5^{\circ} \mathrm{C}$ (water); ${ }^{1} \mathrm{H}$ NMR $\left(300 \mathrm{MHz}, \mathrm{CDCl}_{3}\right): \delta 8.05$ (d, 1H, J $8.8 \mathrm{~Hz}), 7.63$ (dd, 1H, J $17.4 \& 11.0 \mathrm{~Hz}), 7.06$ (d, 1H, J $2.2 \mathrm{~Hz}), 6.87$ (dd, 1H, J 8.8 \& $2.2 \mathrm{~Hz}), 5.64(\mathrm{~d}, 1 \mathrm{H}, J 17.4 \mathrm{~Hz}), 5.37(\mathrm{~d}, 1 \mathrm{H}, J 11.0 \mathrm{~Hz}), 3.89(\mathrm{~s}, 3 \mathrm{H}) ;{ }^{13} \mathrm{C} \mathrm{NMR}(400$ $\left.\mathrm{MHz}, \mathrm{CDCl}_{3}\right): \delta 171.7(\mathrm{C}=\mathrm{O}), 163.3(\mathrm{~s}), 143.4(\mathrm{~s}), 136.5(\mathrm{~d}), 133.8(\mathrm{~d}), 119.4(\mathrm{~s}), 116.7(\mathrm{t})$, 112.9 (d), 112.8 (d), 55.5 (q) ; IR (film, $\mathrm{CHCl}_{3}$ ) : $v_{\max }$ 3428, 2680, 2358, 2341, 1641, 1560, 1295, $1234 \mathrm{~cm}^{-1}$. ES-HRMS: Found 177.0544, $\mathrm{C}_{10} \mathrm{H}_{9} \mathrm{O}_{3}$ requires 177.0552 [M-H] $]^{-}$. Anal. Calcd. for $\mathrm{C}_{10} \mathrm{H}_{10} \mathrm{O}_{3}: \mathrm{C}, 67.41 ; \mathrm{H}$, 5.66. Found: $\mathrm{C}, 67.13$; H, 5.62.

\section{1,6-Di-O-[(4-methoxyphenyl)-diphenylmethyl]-D-mannitol}
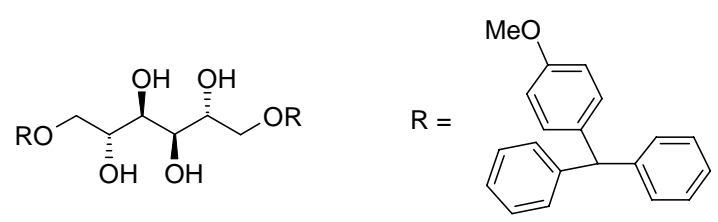

To a solution of D-mannitol 17 (1 g, $5.49 \mathrm{mmol})$ and DMAP (335 mg, $2.75 \mathrm{mmol})$ in dry pyridine $(8 \mathrm{~mL})$ was added dropwise, over $1 \quad \mathrm{~h}, \quad \mathrm{a}$ solution of 4methoxytriphenylmethylchloride $(3.55 \mathrm{~g}, 11.5 \mathrm{mmol})$ in dry pyridine $(12 \mathrm{~mL})$ at $0{ }^{\circ} \mathrm{C}$. The reaction mixture was stirred at room temperature under $\mathrm{N}_{2}$ for $48 \mathrm{~h}$. Ethyl acetate $(100 \mathrm{~mL})$ was added and the mixture washed with satd aq $\mathrm{NH}_{4} \mathrm{Cl}(2 \times 50 \mathrm{~mL})$, brine $(2 \times 50 \mathrm{~mL})$, dried over $\mathrm{Na}_{2} \mathrm{SO}_{4}$, filtered and the solvent removed in vacuo. The residue was purified by chromatography (7:3, cyclohexane-EtOAc) to afford the title compound (3.41 g, $86 \%$, oil); 
$\mathrm{R}_{\mathrm{f}} 0.21\left(7: 3\right.$, cyclohexane-EtOAc); $[\alpha]_{\mathrm{D}}{ }^{20}+0.78^{\circ}$ (c $\left.0.9, \mathrm{CHCl}_{3}\right) ;{ }^{1} \mathrm{H}$ NMR $(300 \mathrm{MHz}$, $\left.\mathrm{CDCl}_{3}\right): \delta 7.40(\mathrm{~m}, 8 \mathrm{H}), 7.25(\mathrm{~m}, 16 \mathrm{H}), 6.78(\mathrm{~d}, 4 \mathrm{H}, J 8.5 \mathrm{~Hz}), 3.91(\mathrm{~m}, 2 \mathrm{H}), 3.77$ (overlapping signals, $8 \mathrm{H}), 3.31(\mathrm{~d}, 4 \mathrm{H}, J 5.1 \mathrm{~Hz}) ;{ }^{13} \mathrm{C} \mathrm{NMR}\left(\mathrm{CDCl}_{3}\right): \delta 158.6(\mathrm{~s}), 144.11$, 144.06, 135.2 (each s), 130.3, 128.3, 127.9, 127.0, 113.2, 86.8, 71.9, 70.9 (each d), $64.8(\mathrm{t})$, 55.2 (q); IR (film, $\mathrm{CHCl}_{3}$ ) : v $v_{\max }$ 3407, 2929, 1639, 1509, 1446, 1251, 1070, 1033, $754 \mathrm{~cm}^{-1}$. ES-HRMS: Found 725.3114, $\mathrm{C}_{46} \mathrm{H}_{45} \mathrm{O}_{8}$ requires $725.3100[\mathrm{M}-\mathrm{H}]^{-}$. Anal. Calcd. for $\mathrm{C}_{46} \mathrm{H}_{46} \mathrm{O}_{8}$ : C, $76.01 ; H, 6.38$. Found: C, $75.62 ; \mathrm{H}, 6.32$.

\section{1,6-Di-O-[(4-methoxyphenyl)-diphenylmethyl]-2,3,4,5-tetra-O-benzyl-D-mannitol}
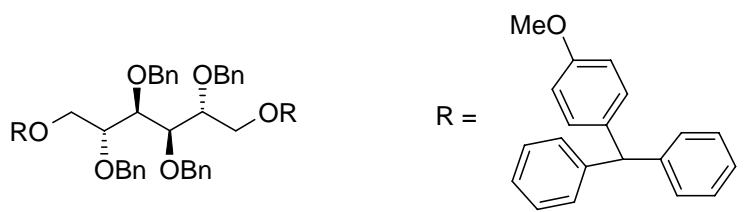

To a solution of 1,6-di-O-[(4-methoxyphenyl)-diphenylmethyl]-D-mannitol (1.57 g, 2.17 mmol, 1 eq.) in dry DMF $(50 \mathrm{~mL})$ was added portionwise sodium hydride (60\% dispersion in oil, $854 \mathrm{mg}, 21.7 \mathrm{mmol})$ at $0{ }^{\circ} \mathrm{C}$, followed by benzyl bromide $(2.58 \mathrm{~mL}, 21.7 \mathrm{mmol})$ dropwise at the same temperature. The reaction mixture was stirred and allowed to attain room temperature for $2 \mathrm{~h}$ under $\mathrm{N}_{2}$. Methanol $(30 \mathrm{~mL})$ was first added at $0{ }^{\circ} \mathrm{C}$ and subsequently EtOAc $(100 \mathrm{~mL})$ and the resulting solution washed with water $(2 \times 50 \mathrm{~mL})$. The organic layer was dried over $\mathrm{MgSO}_{4}$, filtered and the solvent removed in vacuo and the residue purified by chromtography (19:1, cyclohexane-EtOAc) to afford the title compound (2.31 g, $98 \%$, oil); $\mathrm{R}_{\mathrm{f}} 0.30$ (9:1, cyclohexane-EtOAc); $[\alpha]{ }_{\mathrm{D}}^{20}-9$ (c $\left.0.99, \mathrm{CHCl}_{3}\right) ;{ }^{1} \mathrm{H}$ NMR (300 $\left.\mathrm{MHz}, \mathrm{CDCl}_{3}\right): \delta 7.48(\mathrm{~m}, 8 \mathrm{H}), 7.40(\mathrm{~m}, 4 \mathrm{H}), 7.32(\mathrm{~m}, 7 \mathrm{H}), 7.15(\mathrm{~m}, 18 \mathrm{H}), 6.93(\mathrm{~m}, 4 \mathrm{H}), 6.67$ $(\mathrm{d}, 4 \mathrm{H}, J 8.9 \mathrm{~Hz}), 4.74(\mathrm{~d}, 2 \mathrm{H}, J 11.8 \mathrm{~Hz}), 4.37(\mathrm{~m}, 8 \mathrm{H}), 3.85(\mathrm{~m}, 2 \mathrm{H}), 3.70(\mathrm{~s}, 6 \mathrm{H}), 3.66(\mathrm{~m}$, 2H), $3.29(\mathrm{~m}, 2 \mathrm{H}$, dd, J $4.2 \& 10.5 \mathrm{~Hz}) ;{ }^{13} \mathrm{C} \mathrm{NMR}\left(\mathrm{CDCl}_{3}\right): \delta 158.4,144.7,144.5,138.8$, $138.7,135.7$ (each s), 130.5, 128.6, 128.5, 128.3, 127.9, 127.7, 127.4, 127.2, 126.7, 113.0, 
86.3 (each d), 78.6, 73.5 (t), 71.5 (d), 62.0 (t), 55.1 (q); IR (film, $\mathrm{CHCl}_{3}$ ) : $v_{\max }$ 3411, 2358, 1644, $609 \mathrm{~cm}^{-1}$. ES-HRMS: Found 1109.4965, $\mathrm{C}_{74} \mathrm{H}_{70} 0_{8} \mathrm{Na}$ requires $1109.4968[\mathrm{M}+\mathrm{Na}]^{+}$.

\section{2,3,4,5-tetra-O-benzyl-D-mannitol 18}

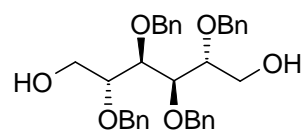

To a solution of 1,6-di-O-[(4-methoxyphenyl)-diphenylmethyl]- 2,3,4,5-tetra-O-benzyl-Dmannitol $(1 \mathrm{~g}, 9.21 \mathrm{mmol})$ in a mixture of $\mathrm{MeOH}$ and $\mathrm{DCM}(10: 3 ; 15 \mathrm{~mL})$ was added dropwise conc $\mathrm{H}_{2} \mathrm{SO}_{4}(20 \mu \mathrm{L})$. The reaction mixture was stirred at room temperature for $19 \mathrm{~h}$ and $\mathrm{NaHCO}_{3}(1 \mathrm{~g})$ was then added. The solution was then filtered, dried over $\mathrm{Na}_{2} \mathrm{SO}_{4}$, filtered and the solvent was removed in vacuo and the residue purified by chromatography (4:1, cyclohexane-EtOAc) to afford 18 (449 $\mathrm{mg}, 90 \%$, colourless oil); $\mathrm{R}_{\mathrm{f}} 0.21$ (7:3,

cyclohexane-EtOAc); $[\alpha]_{\mathrm{D}}{ }^{20} 3\left(\right.$ c $\left._{0.86}, \mathrm{CHCl}_{3}\right) ;{ }^{1} \mathrm{H}$ NMR $\left(300 \mathrm{MHz}, \mathrm{CDCl}_{3}\right): \delta 7.32(\mathrm{~m}$, 20H), 4.77 (d, 2H, J 11.3 Hz), $4.63(\mathrm{~d}, 2 \mathrm{H}, J 11.3 \mathrm{~Hz}), 4.56(\mathrm{~d}, 2 \mathrm{H}, J 11.6 \mathrm{~Hz}), 4.41$ (d, 2H, $J$ $11.6 \mathrm{~Hz}), 3.92(\mathrm{~m}, 4 \mathrm{H}), 3.82(\mathrm{~m}, 2 \mathrm{H}), 3.68(\mathrm{~m}, 2 \mathrm{H}), 2.15(\mathrm{br} \mathrm{s}, 2 \mathrm{H}) ;{ }^{13} \mathrm{C} \mathrm{NMR}\left(\mathrm{CDCl}_{3}\right): \delta$ 138.1, 137.8 (each s), 128.4, 128.3, 127.84, 127.77, 127.6, 79.6, 78.6 (each d), 74.3, 71.4 (each t), 60.3 (d); IR (film, $\mathrm{CHCl}_{3}$ ): $v_{\max }$ 3428, 2962, 2869, 1643, 1396, 1261, 1207, 1099, $734 \mathrm{~cm}^{-1}$. ES-HRMS: Found 541.2571, $\mathrm{C}_{34} \mathrm{H}_{37} \mathrm{O}_{6}$ requires $541.2590[\mathrm{M}-\mathrm{H}]^{-}$.

\section{1-O-Allyl-2,3,4,5-tetra-O-benzyl-D-mannitol 19.}

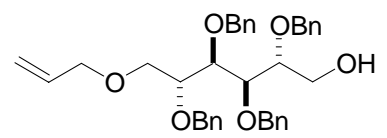

To 18 (202 mg, $0.37 \mathrm{mmol})$ in dry DMF $(12 \mathrm{~mL})$ was added, at $0{ }^{\circ} \mathrm{C}$, sodium hydride $(20 \mathrm{mg}$, $0.52 \mathrm{mmol}$ ). The mixture was stirred for a few min under $\mathrm{N}_{2}$ and allyl bromide $(36 \mu \mathrm{L}, 0.41$ mmol) was then added dropwise at the same temperature. The reaction mixture was allowed 
attain room temperature and stirring continued overnight and $\mathrm{MeOH}$ was then added. The solvent was removed and the residue purified by chromatography $(9: 1$, cyclohexane-EtOAc) to give 19 (149 mg, $69 \%$, colourless oil), $\mathrm{R}_{\mathrm{f}} 0.42\left(7: 3\right.$, cyclohexane-EtOAc); $[\alpha]^{20}{ }_{\mathrm{D}} 4(c$ 0.575, $\left.\mathrm{CHCl}_{3}\right) ;{ }^{1} \mathrm{H}$ NMR $\left(300 \mathrm{MHz}, \mathrm{CDCl}_{3}\right): \delta 7.29$ (m, 20H), 5.90 (ddd, $1 \mathrm{H}, J 17.2,10.4 \&$ $5.6 \mathrm{~Hz}), 5.27(\mathrm{dd}, 1 \mathrm{H}, J 17.2 \& 1.6 \mathrm{~Hz}), 5.16(\mathrm{dd}, 1 \mathrm{H}, J 10.4 \& 1.6 \mathrm{~Hz}), 4.73(\mathrm{~m}, 3 \mathrm{H}), 4.63$ (d, 1H, $J 11.3 \mathrm{~Hz}), 4.62(\mathrm{~d}, 1 \mathrm{H}, J 11.6 \mathrm{~Hz}), 4.52$ (d, 1H, J $11.6 \mathrm{~Hz}), 4.47$ (d, 1H, J $11.8 \mathrm{~Hz}), 4.42$ $(\mathrm{d}, 1 \mathrm{H}, J 11.8 \mathrm{~Hz}), 4.00-3.75(\mathrm{~m}, 7 \mathrm{H}), 3.68(\mathrm{~m}, 2 \mathrm{H}), 2.17(\mathrm{t}, 1 \mathrm{H}, J 6.2 \mathrm{~Hz}) ;{ }^{13} \mathrm{C} \mathrm{NMR}$ $\left(\mathrm{CDCl}_{3}\right): \delta 138.5,138.43,138.39,138.2$ (each s), 134.7, 128.4, 128.31, 128.28, 128.27, $127.88,127.87,127.71,127.70,127.65,127.54,127.52]$ (each d), $117.0(\mathrm{t}), 79.8,79.1,79.0$ 78.9, 74.6 (each d), 74.1, 72.2, 71.9, 71.4 (each t), 69.3, 60.6 (each d); IR (film, $\mathrm{CHCl}_{3}$ ): $v_{\max }$ $3471,3085,3064,3029,2921,2867,2358,2341,1756,1496,1454,1394,1259,1207,1099$, 927, 804, 738, 696, $584 \mathrm{~cm}^{-1}$. ES-HRMS: Found 581.2879, $\mathrm{C}_{37} \mathrm{H}_{41} 0_{6}$ requires 581.2903 [M$\mathrm{H}^{-}$. Anal. Calcd. for $\mathrm{C}_{37} \mathrm{H}_{42} \mathrm{O}_{6}$ : C, 76.26; H, 7.26. Found: C, 75.96; H, 7.26. A small amount of 1,6-di-O-allyl-2,3,4,5-tetra-O-benzyl-D-mannitol was also isolated; $[\alpha]^{20} 7$ (c 0.765 , $\left.\mathrm{CHCl}_{3}\right) ;{ }^{1} \mathrm{H}$ NMR $\left(300 \mathrm{MHz}, \mathrm{CDCl}_{3}\right): \delta 7.28$ (m, 20H), 5.88 (ddd, $1 \mathrm{H}, J$ 17.2, $10.4 \& 5.4 \mathrm{~Hz}$ ), $5.26(\mathrm{dd}, 1 \mathrm{H}, J 17.2 \& 0.8 \mathrm{~Hz}), 5.14(\mathrm{dd}, 1 \mathrm{H}, J 10.4 \& 0.8 \mathrm{~Hz}), 4.72(\mathrm{~d}, 2 \mathrm{H}, J 11.3 \mathrm{~Hz}), 4.69$ (d, 2H, J 11.7 Hz), 4.61 (d, 2H, J $11.3 \mathrm{~Hz}), 4.48$ (d, 2H, J $11.7 \mathrm{~Hz}), 3.96(\mathrm{~m}, 6 \mathrm{H}), 3.83$ (m, 4H), $3.83(\mathrm{dd}, 2 \mathrm{H}, J 10.8 \& 5.4 \mathrm{~Hz}) ;{ }^{13} \mathrm{C} \mathrm{NMR}\left(\mathrm{CDCl}_{3}\right): \delta 138.7$ (s), $134.8(\mathrm{~s}), 128.22$, 128.16, 127.8, 127.5, 127.3 (each d), $116.8(\mathrm{t}), 79.3,79.1,74.2($ each d), 72.2, $71.8(\mathrm{t}), 69.6$ (d); IR (film, $\mathrm{CHCl}_{3}$ ): $v_{\max }$ 2609, 2096, 1641, $1483 \mathrm{~cm}^{-1}$. ES-HRMS: Found 623.3357, $\mathrm{C}_{40} \mathrm{H}_{47} \mathrm{O}_{6}$ requires $623.3373[\mathrm{M}+\mathrm{H}]^{+}$. 


\section{1,6-Di-O-[(4-methoxyphenyl)-diphenylmethyl]-D-glucitol}
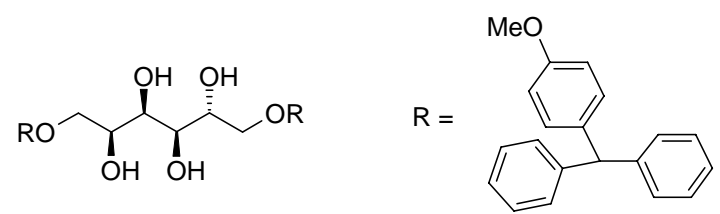

The reaction of D-sorbitol (1.05 g, $5.79 \mathrm{mmol})$, DMAP (354 mg, $2.90 \mathrm{mmol})$ and (4methoxyphenyl)-diphenyl-methylchloride $(3.76 \mathrm{~g}, 12.2 \mathrm{mmol})$ in dry pyridine $(10 \mathrm{~mL})$ gave the title compound $\left(3.48 \mathrm{~g}, 83 \%\right.$, oil); $\mathrm{R}_{\mathrm{f}} 0.36$ (7:3, cyclohexane-EtOAc); $[\alpha]_{\mathrm{D}}{ }^{20}-9^{\circ}$ (c 0.92 , $\left.\mathrm{CHCl}_{3}\right) ;{ }^{1} \mathrm{H}$ NMR $\left(600 \mathrm{MHz}, \mathrm{CDCl}_{3}\right): \delta 7.40(\mathrm{~m}, 8 \mathrm{H}), 7.23(\mathrm{~m}, 16 \mathrm{H}), 6.77(\mathrm{dd}, 4 \mathrm{H}, J 9.0 \&$ $2.8 \mathrm{~Hz}), 3.88(\mathrm{~m}, 2 \mathrm{H}), 3.84(\mathrm{~m}, 1 \mathrm{H}), 3.74(\mathrm{~s}, 3 \mathrm{H}), 3.73(\mathrm{~s}, 3 \mathrm{H}), 3.64(\mathrm{dd}, 1 \mathrm{H}, J 6.5$ \& $1.0 \mathrm{~Hz})$, $3.31(\mathrm{~m}, 3 \mathrm{H}), 3.21(\mathrm{dd}, 1 \mathrm{H}, J 9.8 \& 5.9 \mathrm{~Hz}), 3.13$ (br s, 3H), $2.92($ br s, $1 \mathrm{H}) ;{ }^{13} \mathrm{C} \mathrm{NMR}$ $\left(\mathrm{CDCl}_{3}\right): \delta 158.6,144.16,144.15,144.11,144.03,135.23,135.21$ (each s), 130.27, 130.23 $\left(\mathrm{C}_{\mathrm{c}}\right), 128.31,128.26,127.9,127.0,113.2,86.7,73.7,73.0,71.5,69.9$ (each d), 64.9 (t), 64.8 (t), 55.2 (q), 55.1 (q); IR (film, $\mathrm{CHCl}_{3}$ ): $v_{\max }$ 3438, 3058, 3033, 2931, 2836, 1608, 1509, 1446, 1251, 1180, 1076, 1033, 910, 833, 647, $588 \mathrm{~cm}^{-1}$. ES-HRMS: Found 749.3111, $\mathrm{C}_{46} \mathrm{H}_{46} \mathrm{O}_{8} \mathrm{Na}$ requires $749.3090[\mathrm{M}+\mathrm{Na}]^{+}$.

\section{1,6-Di-O-[(4-methoxyphenyl)-diphenylmethyl]- 2,3,4,5-tetra-O-benzyl-D-glucitol}
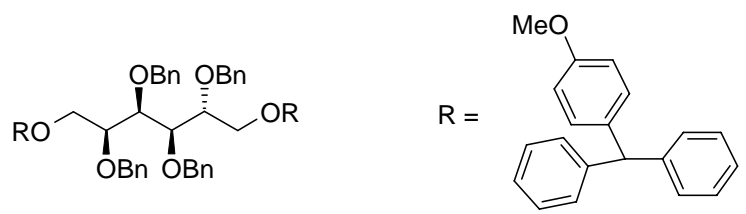

The benzylation of 1,6-di-O-[(4-methoxyphenyl)-diphenylmethyl]-D-glucitol (3.40 g, 4.68 mmol) as described for the corresponding mannitol derivative gave the title compound (4.34 g, $85 \%$, oil $) ; \mathrm{R}_{\mathrm{f}} 0.40$ (9:1, cyclohexane-EtOAc); $[\alpha]^{20}{ }_{\mathrm{D}}+3$ (c $\left.0.5, \mathrm{CHCl}_{3}\right) ;{ }^{1} \mathrm{H}$ NMR (500 $\left.\mathrm{MHz}, \mathrm{CDCl}_{3}\right): \delta 7.44(\mathrm{~m}, 6 \mathrm{H}), 7.20(\mathrm{~m}, 36 \mathrm{H}), 6.80(\mathrm{~m}, 2 \mathrm{H}), 6.68(\mathrm{~m}, 4 \mathrm{H}), 4.71(\mathrm{~d}, 1 \mathrm{H}, J 11.7$ Hz), 4.69 (d, 1H, J 11.6 Hz), 4.60 (d, 1H, J $11.6 \mathrm{~Hz}), 4.56$ (d, 1H, J 11.4 Hz), 4.54 (d, 1H, J 
$11.4 \mathrm{~Hz}), 4.38$ (d, 1H, J 11.7 Hz), 4.33 (d, 1H, J 11.3 Hz), 4.15 (d, 1H, J 11.3 Hz), 4.03 (1H, dd, J 5.5 \& $4.5 \mathrm{~Hz}), 3.95(1 \mathrm{H}$, dd, J 6.2 \& $4.5 \mathrm{~Hz}), 3.89(1 \mathrm{H}, \mathrm{dd}, \mathrm{J} 8.5$ \& $5.5 \mathrm{~Hz}), 3.84$ (m, 1H), $3.70(\mathrm{~s}, 3 \mathrm{H}), 3.69$ (s, 3H), $3.55(\mathrm{dd}, 1 \mathrm{H}, J 10.2 \& 1.3 \mathrm{~Hz}), 3.41(\mathrm{dd}, 1 \mathrm{H}, J 10.2 \& 2.9$ $\mathrm{Hz}), 3.32(\mathrm{dd}, 1 \mathrm{H}, J 10.2 \& 5.3 \mathrm{~Hz}), 3.25(\mathrm{dd}, 1 \mathrm{H}, J 10.2 \& 5.5 \mathrm{~Hz}) ;{ }^{13} \mathrm{C} \mathrm{NMR}\left(\mathrm{CDCl}_{3}\right): \delta$ $158.44,158.40,144.6,144.5,138.85,138.84,138.77,138.52$ (each s), 135.7, 135.6, 130.47, $130.46,128.59,128.57,128.56,128.52,128.24,128.22,128.1,127.90,127.88,127.73$, $127.70,127.69,127.66,127.4,127.18,127.16,127.0,126.75,126.73,126.71,126.70,113.0$, 79.8, 79.4, 79.1, 78.4 (each d), 74.4, 73.6, 72.9, 72.0, 63.5, 62.8 (each t), 55.1 (q); IR (film, $\left.\mathrm{CHCl}_{3}\right): v_{\max } 3085,3062,3029,2962,2927,1606,1508,1448,1255,1178,1087,1029,910$, 804, 730, 700, $638 \mathrm{~cm}^{-1}$. ES-HRMS: Found 1109.4985, $\mathrm{C}_{74} \mathrm{H}_{70} 0_{8} \mathrm{Na}$ requires 1109.4968 $[\mathrm{M}+\mathrm{Na}]^{+}$.

\section{2,3,4,5-Tetra-O-benzyl-D-glucitol}

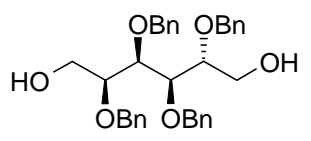

Removal of the MMTr groups from 1,6-di-O-[(4-methoxyphenyl)-diphenylmethyl]- 2,3,4,5tetra-O-benzyl-D-glucitol $(4.12 \mathrm{~g}, 3.79 \mathrm{mmol})$ as described for the related mannitol derivative afforded the title compound (1.04 g, $47 \%$, colourless oil) ; $\mathrm{R}_{\mathrm{f}} 0.27$ (7:3, cyclohexane-EtOAc); $[\alpha]{ }_{\mathrm{D}}^{20}+1.9\left(\mathrm{c} 1.05, \mathrm{CHCl}_{3}\right) ;{ }^{1} \mathrm{H}$ NMR $\left(300 \mathrm{MHz}, \mathrm{CDCl}_{3}\right): \delta 7.29(\mathrm{~m}, 20 \mathrm{H}), 4.70(\mathrm{~m}, 6 \mathrm{H})$, $4.51(\mathrm{~d}, 1 \mathrm{H}, J 11.7 \mathrm{~Hz}), 4.39$ (d, 1H, J $11.7 \mathrm{~Hz}), 3.94$ (t, 1H, J 4.7 Hz), 3.78 (m, 6H), 3.56 (m, 1H), 2.35 (t, 1H, J $6.0 \mathrm{~Hz}), 2.13(\mathrm{t}, 1 \mathrm{H}, J 4.9 \mathrm{~Hz}) ;{ }^{13} \mathrm{C} \mathrm{NMR}\left(400 \mathrm{MHz}, \mathrm{CDCl}_{3}\right): \delta 138.0(\mathrm{~s})$, $128.4,128.3,128.2,128.0,127.9,127.7,127.5,79.6,79.3,78.9,78.3$ (each d), 74.5, 74.0, 72.7, 71.5, 61.6, 60.6 (each t); IR (film, $\mathrm{CHCl}_{3}$ ): $v_{\max }$ 3421, 3062, 3029, 2927, 2875, 1496, 1454, 1209, 1093, 1066, 802, 736, $698 \mathrm{~cm}^{-1}$. ES-HRMS: Found 543.2739, $\mathrm{C}_{34} \mathrm{H}_{39} 0_{6}$ requires $543.2747[\mathrm{M}+\mathrm{H}]^{+}$. 


\section{1-O-Allyl-2,3,4,5-tetra-O-benzyl-D-glucitol 20 and 6-O-allyl-2,3,4,5-tetra- $O$-benzyl-D-} glucitol 21

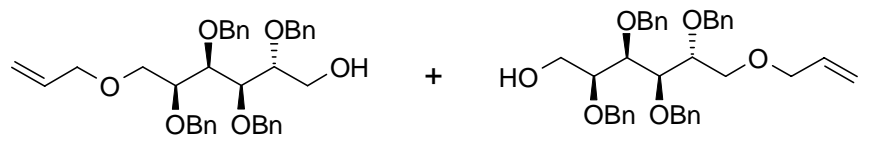

The allylation of 2,3,4,5-tetra-O-benzyl-D-glucitol $(940 \mathrm{mg}, 1.73 \mathrm{mmol})$ as described for 2,3,4,5-tetra-O-benzyl-D-mannitol gave a 1:1 mixture of 20 and 21 (749 mg, $74 \%$, colourless oil) ; $\mathrm{R}_{\mathrm{f}} 0.56$ (1:1, cyclohexane-EtOAc); ${ }^{1} \mathrm{H}$ NMR (400 MHz, $\left.\mathrm{CDCl}_{3}\right): \delta 7.29(\mathrm{~m}, 40 \mathrm{H}), 5.87$ (m, 2H), $5.21(\mathrm{~m}, 4 \mathrm{H}), 4.70(\mathrm{~m}, 12 \mathrm{H}), 4.44(\mathrm{~m}, 4 \mathrm{H}), 3.98(\mathrm{~m}, 4 \mathrm{H}), 3.83(\mathrm{~m}, 9 \mathrm{H}), 3.67(\mathrm{~m}$, 5H), $3.52(\mathrm{~m}, 2 \mathrm{H}), 2.29(\mathrm{~m}, 1 \mathrm{H}), 2.05(\mathrm{~m}, 1 \mathrm{H}) ;{ }^{13} \mathrm{C} \mathrm{NMR}\left(\mathrm{CDCl}_{3}\right): \delta 138.6,138.43,138.36$, $138.33,138.28,138.25,138.17$ (each s), 134.74, 134.67, 128.4, 128.31, 128.30, 128.27, $128.24,128.22,128.05,128.02,127.98,127.9,127.7,127.61,127.59,127.57,127.54$ (each d), 116.9 (t), 79.8, 79.3, 79.0, 78.9, 78.6, 78.3, 74.7, 74.6 (each d), 74.4, 73.8, 72.8, 72.7, 72.2, 72.1, 72.0, 71.4 (each t), 70.1, 69.7, 61.8, 60.9; IR (film, $\mathrm{CHCl}_{3}$ ): $v_{\max }$ 3475, 3062, 3029, 2923, 2871, 1496, 1454, 1349, 1259, 1209, 1089, 1027, 914, 806, 732, 698, $576 \mathrm{~cm}^{-1} . \mathrm{ES}-$ HRMS: Found 583.3068, $\mathrm{C}_{37} \mathrm{H}_{43} \mathrm{O}_{6}$ requires 583.3060 [M+H] $]^{+}$1,6-Di-O-allyl-2,3,4,5-tetra-Obenzyl-D-glucitol was also obtained; ${ }^{1} \mathrm{H}$ NMR $\left(300 \mathrm{MHz}, \mathrm{CDCl}_{3}\right): \delta 7.28(\mathrm{~m}, 20 \mathrm{H}), 5.86(\mathrm{~m}$, 2H), $5.26(\mathrm{~m}, 2 \mathrm{H}), 5.14(\mathrm{~m}, 2 \mathrm{H}), 4.68(\mathrm{~m}, 7 \mathrm{H}), 4.44(\mathrm{~d}, 1 \mathrm{H}, J 11.6 \mathrm{~Hz}), 3.98(\mathrm{~m}, 3 \mathrm{H}), 3.84$ $(\mathrm{m}, 6 \mathrm{H}), 3.67(\mathrm{dd}, 1 \mathrm{H}, J 11.1 \& 6.5 \mathrm{~Hz}), 3.58(\mathrm{dd}, 1 \mathrm{H}, J 10.1 \& 2.4 \mathrm{~Hz}), 3.48(\mathrm{dd}, 1 \mathrm{H}, J 10.1$ \& $4.5 \mathrm{~Hz}) ;{ }^{13} \mathrm{C}$ NMR $\left(\mathrm{CDCl}_{3}\right): \delta 138.7,138.6$ (each s), 134.8, 134.7, 128.1, 128.0, 127.9, 127.42, 127.39, 127.34, 127.27 (each d), 116.7, 116.6 (each t), 79.3, 79.1, 79.07, 78.6, 74.7, 74.0 (each d), 72.8, 72.1, 72.0, 71.9, 70.4, 69.6 (each t); IR (film, $\mathrm{CHCl}_{3}$ ): $v_{\max }$ 3062, 3031, 2917, 2869, 1724, 1602, 1454, 1268, 1093, 1070, 1027, 925, 696, 671, $572 \mathrm{~cm}^{-1}$. ES-HRMS: Found 645.3163, $\mathrm{C}_{40} \mathrm{H}_{46} \mathrm{O}_{6} \mathrm{Na}$ requires $645.3192[\mathrm{M}+\mathrm{Na}]^{+}$. 


\section{1,6-Di-O-pivaloyl-D-mannitol}

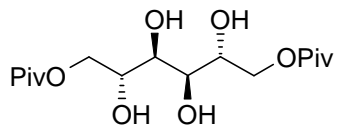

To a solution of D-mannitol $17(1 \mathrm{~g}, 5.49 \mathrm{mmol})$ in pyridine $(8 \mathrm{~mL})$ at $0^{\circ} \mathrm{C}$ under $\mathrm{N}_{2}$ was added dropwise trimethylacetyl chloride $(1.35 \mathrm{~mL}, 11.0 \mathrm{mmol})$ over $20 \mathrm{~min}$. The reaction mixture was allowed to attain room temp and stirred for $16 \mathrm{~h}$ and the solvent removed in vacuo. The residue was dissolved in EtOAc $(50 \mathrm{~mL})$, washed with $\mathrm{HCl}(2 \mathrm{~N}, 10 \mathrm{~mL})$, $\mathrm{NaHCO}_{3}(10 \mathrm{~mL})$, brine $(10 \mathrm{~mL})$ and dried over $\mathrm{Na}_{2} \mathrm{SO}_{4}$. After filtration, the solvent was removed and the residue purified by chromatography $\left(39: 1, \mathrm{CH}_{2} \mathrm{Cl}_{2}-\mathrm{MeOH}\right)$ to give the title compound ( $1.29 \mathrm{~g}, 60 \%$, white solid); $\mathrm{R}_{\mathrm{f}} 0.30$ (3:1 cyclohexane-EtOAc); mp $140.5^{\circ} \mathrm{C}$ (water); $\left[\alpha{ }^{20}{ }_{\mathrm{D}}+3.8\left(\mathrm{c} 0.545, \mathrm{CH}_{3} \mathrm{OH}\right) ;{ }^{1} \mathrm{H}\right.$ NMR $(300 \mathrm{MHz}, \mathrm{MeOD}): \delta 4.36$ (dd, 2H, $J 11.5 \& 2.6$ Hz), $4.16(\mathrm{dd}, 2 \mathrm{H}, J 11.5 \& 5.9 \mathrm{~Hz}), 3.88$ (ddd, 2H, J 8.7, $5.9 \& 2.6 \mathrm{~Hz}), 3.81$ (d, 2H, $J 8.7$ $\mathrm{Hz}), 1.22(\mathrm{~s}, 18 \mathrm{H}) ;{ }^{13} \mathrm{C}$ NMR $(\mathrm{MeOD}): \delta 180.5(2 \times \mathrm{C}=\mathrm{O}), 70.6(\mathrm{~d}), 70.5(\mathrm{~d}), 68.0(\mathrm{t}), 40.0$ (s), 27.6 (q); IR (film, $\mathrm{CH}_{3} \mathrm{OH}$ ): $v_{\max }$ 3401, 2630, 2096, 1639, 1294, 1203, 1108, $1018 \mathrm{~cm}^{-1}$. ES-HRMS: Found 373.1845, $\mathrm{C}_{16} \mathrm{H}_{30} \mathrm{O}_{8} \mathrm{Na}$ requires $373.1838[\mathrm{M}+\mathrm{Na}]^{+}$. Anal. Calcd. for $\mathrm{C}_{16} \mathrm{H}_{30} \mathrm{O}_{8}:$ C, $54.84 ; \mathrm{H}, 8.63$. Found: C, $54.73 ; \mathrm{H}, 8.77$. The 1,2,6-tri-O-pivaloylated-Dmannitol derivative was also obtained $\left(295 \mathrm{mg}, 11 \%\right.$, white solid); $\mathrm{R}_{\mathrm{f}} 0.60$ (3:1, cyclohexaneEtOAc)); $[\alpha]^{20}{ }_{\mathrm{D}}+4.1\left(\right.$ c $\left.0.6, \mathrm{CH}_{3} \mathrm{OH}\right) ; \mathrm{mp} \sim 136.5-140{ }^{\circ} \mathrm{C}$ (water); ${ }^{1} \mathrm{H}$ NMR $(300 \mathrm{MHz}$, MeOD): $\delta 5.10$ (ddd, 1H, J 9.3, $5.2 \& 2.3 \mathrm{~Hz}), 4.65$ (dd, 1H, $J 12.1 \& 2.3 \mathrm{~Hz}), 4.35$ (dd, $1 \mathrm{H}, J$ $11.6 \& 2.7 \mathrm{~Hz}), 4.22(\mathrm{dd}, 1 \mathrm{H}, J 12.1 \& 5.2 \mathrm{~Hz}), 4.15(\mathrm{dd}, 1 \mathrm{H}, J 11.6 \& 5.3 \mathrm{~Hz}), 4.11(\mathrm{dd}, 1 \mathrm{H}$, $J 9.3 \& 1.0 \mathrm{~Hz}), 3.90(\mathrm{ddd}, 1 \mathrm{H}, J 9.0,5.3 \& 2.6 \mathrm{~Hz}), 3.51(\mathrm{dd}, 1 \mathrm{H}, J 9.2 \& 1.0 \mathrm{~Hz}), 1.24(\mathrm{~s}$, 9H), $1.23(\mathrm{~s}, 9 \mathrm{H}), 1.22(\mathrm{~s}, 9 \mathrm{H}) ;{ }^{13} \mathrm{C}$ NMR (MeOD): $\delta 180.5,179.8,178.9($ each $\mathrm{C}=\mathrm{O}), 72.4$, 70.4, 70.1 (each d), $68.6(\mathrm{t}), 67.7$ (d), 64.2 (t), 40.0 (s), 39.92 (q), 39.87 (q), 27.6 (q); IR (film, 
$\left.\mathrm{CH}_{3} \mathrm{OH}\right): v_{\max } 3517,3446,3417,2960,2910,2603,2543,1702,1294,1197,1103,1083$, 1025, $914 \mathrm{~cm}^{-1}$. ES-HRMS: Found 457.2436, $\mathrm{C}_{21} \mathrm{H}_{38} \mathrm{O}_{9} \mathrm{Na}$ requires $457.2414[\mathrm{M}+\mathrm{Na}]^{+}$.

\section{1,6-Di-O-pivaloyl-2,3,4,5-tetra-O-methoxymethyl-D-mannitol}

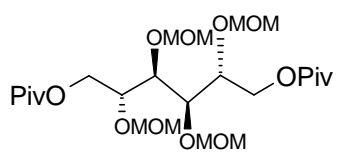

To a suspension of 1,6-di-O-pivaloyl-D-mannitol $(0.71 \mathrm{~g}, 2.03 \mathrm{mmol})$ and activated molecular sieves $4 \AA$ ( $1 \mathrm{~g})$ in dry dichloromethane $(40 \mathrm{~mL})$ was added $\mathrm{iPr}_{2} \mathrm{NEt}(5.69 \mathrm{~mL}, 32.5 \mathrm{mmol})$ at room temperature under $\mathrm{N}_{2}$. The mixture was stirred for $15 \mathrm{~min}$ and methoxymethyl chloride (2.47 $\mathrm{mL}, 32.5 \mathrm{mmol})$ was then added and the stirring continued for 3 days. Diethyl ether $(500 \mathrm{~mL})$ was added to the mixture which was then filtered and the solvent removed in vacuo. The residue was purified by chromatography (7:3, cyclohexane-EtOAc) to afford the title compound $\left(1.0 \mathrm{~g}, 94 \%\right.$, colourless oil); $\mathrm{R}_{\mathrm{f}} 0.46\left(1: 1\right.$, cyclohexane-EtOAc); $[\alpha]^{20}{ }_{\mathrm{D}}+33(\mathrm{c}$ 0.625, $\left.\mathrm{CHCl}_{3}\right) ;{ }^{1} \mathrm{H}$ NMR (300 MHz, $\left.\mathrm{CDCl}_{3}\right): \delta 4.72(\mathrm{~s}, 8 \mathrm{H}), 4.46(\mathrm{~d}, 2 \mathrm{H}, J 11.9 \mathrm{~Hz}), 4.15(\mathrm{~m}$, 2H), $3.94(\mathrm{~s}, 4 \mathrm{H}), 3.38(\mathrm{~s}, 6 \mathrm{H}), 3.37(\mathrm{~s}, 6 \mathrm{H}), 1.20(\mathrm{~s}, 18 \mathrm{H}) ;{ }^{13} \mathrm{C} \mathrm{NMR}\left(\mathrm{CDCl}_{3}\right): \delta 178.2(\mathrm{~s})$, 98.0, 96.7 (each t), 77.0, 76.4, 64.0 (each d), 56.2, 55.8 (each t), 38.8 (s), 27.2 (q); IR (film, $\left.\mathrm{CHCl}_{3}\right): v_{\max } 2962,2939,2896,2360,2329,1729,1644,1284,1153,1106,1031,919 \mathrm{~cm}^{-1}$. ES-HRMS: Found 549.2878, $\mathrm{C}_{24} \mathrm{H}_{46} \mathrm{O}_{12} \mathrm{Na}$ requires 549.2887 [M+Na] $]^{+}$

\section{2,3,4,5-Tetra-O-methoxymethyl-D-mannitol}

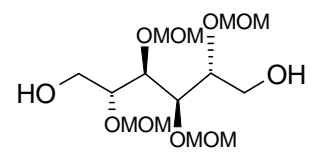

To a solution of 1,6-di-O-pivaloyl-2,3,4,5-tetra-O-methoxymethyl-D-mannitol (561 mg, 1.07 mmol) in $\mathrm{MeOH}(10 \mathrm{~mL})$ cooled on ice was added freshly prepared $\mathrm{NaOMe}$ in $\mathrm{MeOH}(1 \mathrm{M}$, $0.533 \mathrm{~mL}, 0.53 \mathrm{mmol})$. The reaction mixture was allowed to stir at room temperature for a 
few minutes and then heated at $40{ }^{\circ} \mathrm{C}$ for $24 \mathrm{~h}$. Water $(5 \mathrm{~mL})$ was added to the mixture which was then extracted using dichloromethane $(3 \times 30 \mathrm{~mL})$. The organic layer was dried over $\mathrm{MgSO}_{4}$, filtered and the solvent removed in vacuo. The residue was purified by chromatography (1:9, cyclohexane-EtOAc) to afford the title compound (370 $\mathrm{mg}, 97 \%$, colourless oil); $\mathrm{R}_{\mathrm{f}} 0.34$ (1:9, cyclohexane-EtOAc); $[\alpha]{ }_{\mathrm{D}}^{20}+63\left(\right.$ c $\left.0.57, \mathrm{CHCl}_{3}\right) ;{ }^{1} \mathrm{H} \mathrm{NMR}$ (300 MHz, MeOD): $\delta 4.73(\mathrm{~m}, 8 \mathrm{H}), 3.93(\mathrm{~m}, 2 \mathrm{H}), 3.80(\mathrm{~m}, 4 \mathrm{H}), 3.68(\mathrm{dd}, 2 \mathrm{H}, J 10.8 \& 4.9$ $\mathrm{Hz}), 3.39$ (d, 12H, J 2.9 Hz); ${ }^{13} \mathrm{C} \mathrm{NMR}\left(\mathrm{CDCl}_{3}\right): \delta 98.7,96.9$ (each t), 80.4, 77.3 (each d), 61.9 (t), 56.5, 55.9 (each q); IR (film, $\mathrm{CHCl}_{3}$ ): $v_{\max }$ 3438, 2588, 2360, 2341, 2121, 1641, 1380, $1029 \mathrm{~cm}^{-1}$; ES-HRMS: Found 381.1740, $\mathrm{C}_{14} \mathrm{H}_{30} 0_{10} \mathrm{Na}$ requires $381.1737[\mathrm{M}+\mathrm{Na}]^{+}$.

\section{1-O-Allyl-2,3,4,5-tetra-O-methoxymethyl-D-mannitol 22}

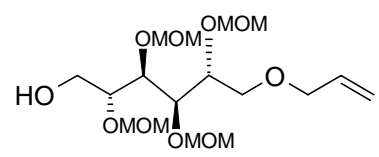

To a solution of 2,3,4,5-tetra-O-methoxymethyl-D-mannitol $(267 \mathrm{mg}, 0.75 \mathrm{mmol})$ in dry DMF $(25 \mathrm{~mL})$ was added portionwise at $0{ }^{\circ} \mathrm{C}$ sodium hydride $(41 \mathrm{mg}, 1.04 \mathrm{mmol})$. The suspension was stirred for a few minutes and allyl bromide added dropwise at the same temperature. The reaction mixture was stirred overnight and then quenched with $\mathrm{MeOH}$. The solvent was removed and the residue purified by chromatography $(3: 2$, cyclohexane-EtOAc) to afford 22 (235 mg, $79 \%$, colourless oil); $\mathrm{R}_{\mathrm{f}} 0.45$ (1:9, cyclohexane-EtOAc); $[\alpha]^{20}{ }_{\mathrm{D}}+46(c$ 0.955, $\left.\mathrm{CHCl}_{3}\right) ;{ }^{1} \mathrm{H} \mathrm{NMR}\left(600 \mathrm{MHz}, \mathrm{CDCl}_{3}\right): \delta 5.91$ (ddd, $1 \mathrm{H}, J 16.1,10.9 \& 5.7 \mathrm{~Hz}$ ), 5.29 (m, 1H), $5.18(\mathrm{~m}, 1 \mathrm{H}), 4.77(\mathrm{~m}, 8 \mathrm{H}), 4.01(\mathrm{~m}, 2 \mathrm{H}), 3.96,3.89,3.76$ and $3.60(\mathrm{~ms}, 8 \mathrm{H}), 3.42$ $(\mathrm{m}, 12 \mathrm{H}), 3.30(\mathrm{br} \mathrm{s}, 1 \mathrm{H}) ;{ }^{13} \mathrm{C} \mathrm{NMR}\left(\mathrm{CDCl}_{3}\right): \delta 134.5$ (d), 116.9, 98.4, 97.9, 96.8, 96.3 (each t), 80.3, 77.3, 77.2, 76.7 (each d), 72.1, 69.4, 62.0 (each t), 56.1, 56.0, 55.6, 55.5 (each q); IR (film, $\mathrm{CHCl}_{3}$ ): $v_{\max }$ 3444, 2958, 2900, 2825, 2530, 2102, 1644, 1469, 1446, 1213, 1151, 1101, 1029, $917 \mathrm{~cm}^{-1}$. ES-HRMS: Found 421.2047, $\mathrm{C}_{17} \mathrm{H}_{34} 0_{10} \mathrm{Na}$ requires $421.2050[\mathrm{M}+\mathrm{Na}]^{+}$. 
1-O-Allyl-6-O-(2-vinyl-benzoyl)-2,3,4,5-tetra-O-benzyl-D-mannitol 23a. To a solution of 19 (98 mg, $0.17 \mathrm{mmol}), 14 \mathrm{a}(27 \mathrm{mg}, 0.18 \mathrm{mmol})$ and triphenylphosphine ( $89 \mathrm{mg}, 0.34 \mathrm{mmol})$ in dry toluene $(2 \mathrm{~mL})$ was added dropwise DIAD $(66 \mu \mathrm{L}, 0.34 \mathrm{mmol})$ at room temperature under $\mathrm{N}_{2}$. The reaction mixture was left stirring for 40 min before being diluted with EtOAc $(10 \mathrm{~mL})$. The solution was washed with satd aq $\mathrm{NH}_{4} \mathrm{Cl}(2 \times 5 \mathrm{~mL})$, brine $(2 \times 5 \mathrm{~mL})$, dried over $\mathrm{MgSO}_{4}$, filtered and the solvent removed in vacuo. The residue was purified by chromatography (9:1, cyclohexane-EtOAc) to give 23a (104 mg, 81\%, colourless oil); $\mathrm{R}_{\mathrm{f}} 0.34$ (9:1 cyclohexane-EtOAc)); ${ }^{1} \mathrm{H}$ NMR $\left(500 \mathrm{MHz}, \mathrm{CDCl}_{3}\right): \delta 7.81(\mathrm{dd}, 1 \mathrm{H}, J 7.9 \& 1.3 \mathrm{~Hz})$, 7.57 (d, 1H, J 7.6 Hz), 7.47 (td, 1H, J 7.6 \& $1.3 \mathrm{~Hz}), 7.44$ (dd, 1H, J 17.4 \& $11.0 \mathrm{~Hz}), 7.32-$ $7.21(\mathrm{~m}, 21 \mathrm{H}), 5.88(\mathrm{ddt}, 1 \mathrm{H}, J 17.2,10.5 \& 5.6 \mathrm{~Hz}), 5.61(\mathrm{dd}, 1 \mathrm{H}, J 17.4 \& 1.3 \mathrm{~Hz}), 5.26$ (dd, 1H, J 11.0 \& $1.3 \mathrm{~Hz}), 5.25$ (ddd, 1H, J 17.2, 3.3 \& 1.6 Hz), 5.14 (ddd, 1H, J 10.5, 2.9 \& $1.3 \mathrm{~Hz}), 4.87(\mathrm{dd}, 1 \mathrm{H}, J 12.2$ \& $2.6 \mathrm{~Hz}), 4.72(2 \mathrm{x} \mathrm{d}, 2 \mathrm{H}, J 11.5 \mathrm{~Hz}), 4.71(\mathrm{~d}, 1 \mathrm{H}, J 11.8 \mathrm{~Hz})$, $4.64(\mathrm{~m}, 3 \mathrm{H}), 4.46(\mathrm{~m}, 2 \mathrm{H}), 4.44(\mathrm{dd}, 1 \mathrm{H}, J 12.2 \& 5.3 \mathrm{~Hz}), 4.07$ (dd, 1H, J 5.6 \& $4.1 \mathrm{~Hz})$, $4.00(\mathrm{dd}, 1 \mathrm{H}, J 5.7$ \& $4.1 \mathrm{~Hz}), 3.949$ (ddd, 1H, J 5.6, 5.3 \& 2.6 Hz), 3.948 (dd, 1H, J 5.6 \& $1.4 \mathrm{~Hz}), 3.950(\mathrm{dd}, 1 \mathrm{H}, J 5.6 \& 1.4 \mathrm{~Hz}), 3.88(\mathrm{~m}, 1 \mathrm{H}), 3.85(\mathrm{dd}, 1 \mathrm{H}, J 10.4 \& 3.1 \mathrm{~Hz}), 3.67$ $(\mathrm{dd}, 1 \mathrm{H}, J 10.4 \& 4.7 \mathrm{~Hz}) ;{ }^{13} \mathrm{C} \mathrm{NMR}\left(\mathrm{CDCl}_{3}\right): \delta 167.1(\mathrm{C}=\mathrm{O}), 139.7,138.65,138.6,138.4$, 138.1 (each s), 136.0, 134.8, 132.0, 130.3 (each d), 128.8 (s), 128.26, 128.25, 127.8, 127.7, 127.6, 127.53, 127.50, 127.48, 127.43, 127.35, 127.1 (each d), $116.9(\mathrm{t}), 116.4(\mathrm{t}), 79.1,79.0$, 78.9, 78.0 (each d), 74.5, 74.2, 72.2, 71.9, 71.6, 69.3, 63.5 (each t). ES-HRMS: Found 735.3326, $\mathrm{C}_{46} \mathrm{H}_{48} \mathrm{O}_{7} \mathrm{Na}$ requires $735.3298[\mathrm{M}+\mathrm{Na}]^{+}$.

1-O-Allyl-6-O-(5-methoxy-2-vinyl-benzoyl)-2,3,4,5-tetra-O-benzyl-D-mannitol 23b<smiles>C=CCOCC(Cc1ccccc1)[C@H](OCc1ccccc1)[C@H](OCc1ccccc1)[C@H](Cc1ccccc1)OCc1ccccc1</smiles> 
Acid 14b (320 mg, $0.55 \mathrm{mmol}$ and $19(108 \mathrm{mg}, 0.60 \mathrm{mmol})$ were treated as described for 23a and gave 23b (263 mg, 64\%, colourless oil, $\mathrm{R}_{\mathrm{f}} 0.51\left(4: 1\right.$, cyclohexane-EtOAc)); $[\alpha]^{20}{ }_{\mathrm{D}}+18$ (c $\left.0.58, \mathrm{CHCl}_{3}\right) ;{ }^{1} \mathrm{H}$ NMR $\left(500 \mathrm{MHz}, \mathrm{CDCl}_{3}\right): \delta 7.52(\mathrm{~d}, 1 \mathrm{H}, J$ 8.7 Hz), 7.38-7.20 (m, 22H), $7.03(\mathrm{dd}, 1 \mathrm{H}, J 8.6 \& 2.8 \mathrm{~Hz}), 5.87(\mathrm{tdd}, 1 \mathrm{H}, J 17.0,10.8$ \& $5.6 \mathrm{~Hz}), 5.52(\mathrm{dd}, 1 \mathrm{H}, J 17.4$ \& $1.2 \mathrm{~Hz}), 5.25(\mathrm{ddd}, 1 \mathrm{H}, J 17.2,3.1 \& 1.5 \mathrm{~Hz}), 5.16(\mathrm{dd}, 1 \mathrm{H}, J 11.0 \& 1.2 \mathrm{~Hz}), 5.14(\mathrm{~m}, 1 \mathrm{H})$, $4.87(\mathrm{dd}, 1 \mathrm{H}, J 12.2 \& 2.5 \mathrm{~Hz}), 4.71(\mathrm{~m}, 3 \mathrm{H}), 4.64(\mathrm{~m}, 3 \mathrm{H}), 4.48(\mathrm{~d}, 1 \mathrm{H}, J 12.0 \mathrm{~Hz}), 4.46(\mathrm{dd}$, $1 \mathrm{H}, J 12.2 \& 4.4 \mathrm{~Hz}), 4.43(\mathrm{~d}, 1 \mathrm{H}, J 12.0 \mathrm{~Hz}), 4.05(\mathrm{dd}, 1 \mathrm{H}, J 5.4 \& 4.2 \mathrm{~Hz}), 3.98(\mathrm{dd}, 1 \mathrm{H}, J$ $5.5 \& 4.2 \mathrm{~Hz}), 3.95(\mathrm{~m}, 3 \mathrm{H}), 3.86(\mathrm{~m}, 1 \mathrm{H}), 3.84(\mathrm{dd}, 1 \mathrm{H}, J 10.5$ \& $3.2 \mathrm{~Hz}), 3.74(\mathrm{~s}, 3 \mathrm{H}), 3.66$ $(\mathrm{dd}, 1 \mathrm{H}, J 10.4 \& 4.8 \mathrm{~Hz}) ;{ }^{13} \mathrm{C} \mathrm{NMR}\left(\mathrm{CDCl}_{3}\right): \delta 167.1(\mathrm{C}=\mathrm{O}), 158.7,138.62,138.57,138.4$, 138.2 (each s), 135.3 (d), 134.8 (d), 132.1 (s), 129.8 (s), 128.31, 128.27, 128.25, 127.83, 127.82, 127.60, 127.57, 127.51, 127.49, 127.47, 127.43, 118.5 (each d), 116.9 (t), 114.7 (d), $114.6(\mathrm{t}), 79.1,79.0,78.9,78.1$ (each t), 74.5, 74.2, 72.2, 71.9, 71.7, 69.3, 63.8 (each t), 55.4 (q); IR (film, $\mathrm{CHCl}_{3}$ ): $v_{\max }$ 3087, 3064, 3029, 2958, 2923, 2856, 2358, 2339, 1720, 1606, 1494, 1454, 1319, 1286, 1261, 1220, 1110, 1070, 1027, 912, 798, 734, $700 \mathrm{~cm}^{-1}$. ES-HRMS: Found 765.3391, $\mathrm{C}_{47} \mathrm{H}_{50} \mathrm{O}_{8} \mathrm{Na}$ requires $765.3403[\mathrm{M}+\mathrm{Na}]^{+}$.

\section{$4(R), 5(R), 6(R), 7(R)$-Tetra-benzyloxy-3,4,5,6,7,8,10-heptahydro-15-methoxy-[11E]-1H-}

\section{2,9-benzdioxa-cyclotetradecin-1-one $24 b$}

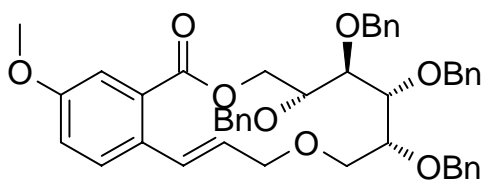

Ring closing metathesis using $\mathbf{2 3 b}$ (90 $\mathrm{mg}, 0.12 \mathrm{mmol}$ ), as described for 24a gave 24b (79 mg, 92\%, colourless oil); $\mathrm{R}_{\mathrm{f}} 0.35$ (4:1 cyclohexane-EtOAc); ${ }^{1} \mathrm{H}$ NMR (600 $\left.\mathrm{MHz}, \mathrm{CDCl}_{3}\right): \delta$ 7.45-7.05 (m, 23H), $7.02(\mathrm{dd}, 1 \mathrm{H}, J 8.6 \& 2.7 \mathrm{~Hz}), 5.98(\mathrm{~d}, 1 \mathrm{H}, J 11.2 \mathrm{~Hz}), 4.97(\mathrm{~m}, 1 \mathrm{H}), 4.86$ (d, 1H, J 9.4 Hz), $4.72(\mathrm{~m}, 3 \mathrm{H}), 4.47$ (m, 4H), 4.32 (d, 2H, J $11.2 \mathrm{~Hz}), 4.26(\mathrm{~m}, 1), 4.17$ (m, 1H), $4.08(\mathrm{~m}, 2 \mathrm{H}), 4.01(\mathrm{~d}, 1 \mathrm{H}, J 7.1 \mathrm{~Hz}), 3.84(\mathrm{~s}, 3 \mathrm{H}), 3.70(\mathrm{~m}, 2 \mathrm{H}) ;{ }^{13} \mathrm{C} \mathrm{NMR}\left(\mathrm{CDCl}_{3}\right): \delta$ 
$168.2(\mathrm{C}=\mathrm{O}), 158.6,139.1,139.0,138.2,137.4$ (each s), 131.9 (d), 130.6, 130.4 (each s), $128.7,128.3,128.2,128.1,127.8,127.7,127.4,127.2,127.2,126.1,118.8,114.8,82.7,78.8$ (each d), 75.5, 74.6, 71.8, 71.3, 69.5, 68.4, 61.1 (each t), 55.4 (q); IR (film, $\mathrm{CHCl}_{3}$ ): $v_{\max }$ $3029,2921,2858,1720,1604,1496,1454,1288,1261,1095,1074,1027,906,865,806,736$, $700 \mathrm{~cm}^{-1}$. ES-HRMS: Found 737.3081, $\mathrm{C}_{45} \mathrm{H}_{46} \mathrm{O}_{8} \mathrm{Na}$ requires $737.3090[\mathrm{M}+\mathrm{Na}]^{+}$.

\section{$4(R), 5(R), 6(R), 7(R)$-Tetra-benzyloxy-3,4,5,6,7,8,10,11,12-nonahydro-15-methoxy-1H-2,9-}

\section{benzdioxa-cyclotetradecin-1-one $9 b$}

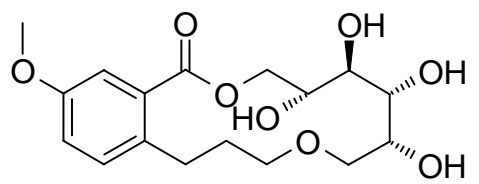

Catalytic hydrogenation of a solution of $\mathbf{2 4 b}(70 \mathrm{mg}, 0.098 \mathrm{mmol})$ as described for 9a afforded 9b (25 mg, 71\%, crystalline solid); $\mathrm{R}_{\mathrm{f}} 0.38\left(9: 1, \mathrm{CH}_{2} \mathrm{Cl}_{2}\right) ; \mathrm{mp} 74{ }^{\circ} \mathrm{C}(\mathrm{MeOH}) ;[\alpha]^{20}$ -12 (c 0.465, $\left.\mathrm{CH}_{3} \mathrm{OH}\right) ;{ }^{1} \mathrm{H} \mathrm{NMR}(600 \mathrm{MHz}, \mathrm{MeOD}): \delta 7.35$ (d, 1H, $\left.J 2.8 \mathrm{~Hz}\right), 7.28(\mathrm{~d}, 1 \mathrm{H}, J$ $8.6 \mathrm{~Hz}), 7.07$ (dd, 1H, J 8.5 \& 2.8 Hz), 4.65 (dd, 1H, J 11.4, $2.1 \mathrm{~Hz}), 4.47$ (dd, 1H, J 7.8 \& 3.4 Hz), $4.44(\mathrm{dd}, 1 \mathrm{H}, J 11.4 \& 4.7 \mathrm{~Hz}), 4.13(\mathrm{dt}, 1 \mathrm{H}, J 6.1 \& 2.8 \mathrm{~Hz}), 4.03(\mathrm{t}, 1 \mathrm{H}, J 3.0 \mathrm{~Hz}), 3.92$ (m, 1H), $3.82(\mathrm{~s}, 3 \mathrm{H}), 3.70(\mathrm{dd}, 1 \mathrm{H}, J 9.4 \& 7.1 \mathrm{~Hz}), 3.55(\mathrm{~m}, 3 \mathrm{H}), 3.12(\mathrm{~m}, 1 \mathrm{H}), 3.01(\mathrm{~m}$, 1H), $1.85(\mathrm{~m}, 2 \mathrm{H}) ;{ }^{13} \mathrm{C}$ NMR (MeOD): $\delta 170.7(\mathrm{C}=\mathrm{O}), 159.0,135.5,132.7$ (each s), 132.6, 119.4, 116.3, 74.0, 72.1 (each d), 71.8, 71.7, 71.0, 67.4 (each t), 55.9 (q), $33.2(\mathrm{t}), 31.4(\mathrm{t})$; IR (film, $\mathrm{CH}_{3} \mathrm{OH}$ ): $v_{\max } 2931,2861,2360,2341,2078,1708,1608,1567,1500,1459,1417$, 1284, 1261, 1226, 1118, 1081, $1035 \mathrm{~cm}^{-1}$. ES-HRMS: Found 379.1385, $\mathrm{C}_{17} \mathrm{H}_{24} \mathrm{O}_{8} \mathrm{Na}$ requires $379.1369[\mathrm{M}+\mathrm{Na}]^{+}$. 


\section{1-O-Allyl-6-O-(4-methoxy-2-vinyl-benzoyl)-2,3,4,5-tetra-O-benzyl-D-mannitol 23c}

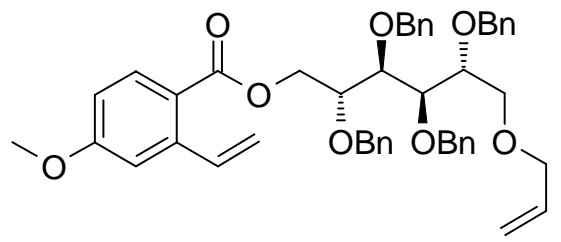

The reaction of $16(135 \mathrm{mg}, 0.23 \mathrm{mmol})$ and $19(50 \mathrm{mg}, 0.28 \mathrm{mmol})$ as described for 23a gave 23c (94 mg, 55\%, colourless oil); $\mathrm{R}_{\mathrm{f}} 0.59$ (7:3 cyclohexane-EtOAc)); ${ }^{1} \mathrm{H}$ NMR (600 $\left.\mathrm{MHz}, \mathrm{CDCl}_{3}\right): \delta 7.85(\mathrm{~d}, 1 \mathrm{H}, J 8.7 \mathrm{~Hz}), 7.53(\mathrm{dd}, 1 \mathrm{H}, J 17.4 \& 10.9 \mathrm{~Hz}), 7.26(\mathrm{~m}, 20 \mathrm{H}), 7.04$ (d, 1H, J 2.6 Hz), 6.77 (dd, 1H, J $8.8 \& 2.6 \mathrm{~Hz}), 5.88$ (ddt, 1H, J 17.2, $10.8 \& 5.6 \mathrm{~Hz}), 5.58$ $(\mathrm{dd}, 1 \mathrm{H}, J 17.4 \& 1.3 \mathrm{~Hz}), 5.28(\mathrm{dd}, 1 \mathrm{H}, J 10.9 \& 1.2 \mathrm{~Hz}), 5.25$ (ddd, $1 \mathrm{H}, J$ 17.2, $3.2 \& 1.6$ Hz), 5.14 (ddd, 1H, J 10.3, $2.7 \& 1.2 \mathrm{~Hz}), 4.82(\mathrm{dd}, 1 \mathrm{H}, J 12.2 \& 2.6 \mathrm{~Hz}), 4.68(\mathrm{~m}, 6 \mathrm{H}), 4.47$ (d, 1H, J $11.8 \mathrm{~Hz}), 4.43$ (d, 1H, J $11.5 \mathrm{~Hz}), 4.42$ (m, 1H), 4.07 (dd, 1H, J 5.6 \& $4.2 \mathrm{~Hz}), 4.00$ $(\mathrm{dd}, 1 \mathrm{H}, J 5.7 \& 4.2 \mathrm{~Hz}), 3.94(\mathrm{~m}, 2 \mathrm{H}), 3.88(\mathrm{~m}, 1 \mathrm{H}), 3.87(\mathrm{~s}, 3 \mathrm{H}), 3.84(\mathrm{dd}, 1 \mathrm{H}, J 10.5 \& 3.1$ $\mathrm{Hz}), 3.67(\mathrm{dd}, 1 \mathrm{H}, J 10.5 \& 4.9 \mathrm{~Hz}) ;{ }^{13} \mathrm{C} \mathrm{NMR}\left(\mathrm{CDCl}_{3}\right): \delta 166.5(\mathrm{C}=\mathrm{O}), 162.5,142.5,138.7$, $138.6,138.5,138.2$ (each s), 136.5, 134.8, 132.8, 128.26, 128.25, 128.24, 128.23, 127.84, $127.83,127.7,127.6,127.49,127.47,127.45,127.4$ (each d, 120.9 (s), 116.9, 116.3 (each t), 112.8, 112.4, 79.1, 79.0, 78.9, 77.9 (each d), 74.5, 74.2, 72.2, 71.8, 71.6, 69.3, 63.2 (each t), 55.4 (q); IR (film, $\mathrm{CHCl}_{3}$ ): $v_{\max }$ 3087, 3062, 3029, 2917, 2869, 2355, 1739, 1712, 1666, 1623, $1600,1563,1525,1496,1454,1321,1257,1236,1205,1095,1027,914,734,698 \mathrm{~cm}^{-1}$. ESHRMS: Found 743.3578, $\mathrm{C}_{47} \mathrm{H}_{51} \mathrm{O}_{8}$ requires $743.3584[\mathrm{M}+\mathrm{H}]^{+}$. 


\section{4(R),5(R),6(R),7(R)-Tetra-benzyloxy-3,4,5,6,7,8,10-heptahydro-14-methoxy-[11E]-1H-}

\section{2,9-benzdioxa-cyclotetradecin-1-one 24c}

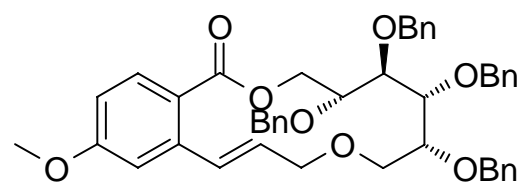

The ring closing metathesis reaction of 23c $(42 \mathrm{mg}, 0.056 \mathrm{mmol})$ as described for 24b gave 24c (25 mg, 63\%, colourless oil); $\mathrm{R}_{\mathrm{f}} 0.24$ (9:1, cyclohexane-EtOAc) as well as recovery of unreacted 23c (14 mg, 33\%). Analytical data for 24c: ${ }^{1} \mathrm{H}$ NMR $\left(500 \mathrm{MHz}, \mathrm{CDCl}_{3}\right): \delta 7.95(\mathrm{~d}$, 1H, J 8.7 Hz), 7.47 (m, 1H), 7.36-7.07 (m, 20H), 6.94 (m, 1H), 6.85 (dd, 1H, J $8.7 \& 2.6 \mathrm{~Hz})$, $6.05(\mathrm{~d}, 1 \mathrm{H}, J 15.6 \mathrm{~Hz}), 4.85(\mathrm{~m}, 2 \mathrm{H}), 4.74(\mathrm{~m}, 1 \mathrm{H}), 4.66(\mathrm{~d}, 1 \mathrm{H}, J 11.3 \mathrm{~Hz}), 4.48$ (m), 4.31 (d, 1H, J $11.4 \mathrm{~Hz}), 4.26(\mathrm{~m}, 3 \mathrm{H}), 4.11(\mathrm{~m}, 2 \mathrm{H}), 4.01(\mathrm{~d}, 1 \mathrm{H}, J 7.5 \mathrm{~Hz}), 3.87(\mathrm{~s}, 3 \mathrm{H}), 3.72(\mathrm{dd}$, $1 \mathrm{H}, J 10.7 \& 4.1 \mathrm{~Hz}), 3.66(\mathrm{~m}, 1 \mathrm{H}) ;{ }^{13} \mathrm{C} \mathrm{NMR}\left(\mathrm{CDCl}_{3}\right): \delta 167.9(\mathrm{C}=\mathrm{O}), 162.6,140.7,139.2$, 139.0, 138.2, 137.6 (each s), 133.9, 132.9, 128.4, 128.21, 128.19, 128.15, 128.0, 127.80, $127.78,127.5,127.3,121.6,112.7,112.6$ (each d), 82.8, 78.9, 77.2, 74.7, 71.8, 71.3, 69.6, 68.8 (each t), 55.4 (q); IR (film, $\mathrm{CHCl}_{3}$ ): $v_{\max }$ 3062, 3029, 2925, 2867, 2360, 2337, 1702, $1600,1562,1529,1496,1454,1429,1371,1267,1122,1027,777,738,698,655,615,592$ $\mathrm{cm}^{-1}$. ES-HRMS: Found 715.3250, $\mathrm{C}_{45} \mathrm{H}_{47} \mathrm{O}_{8}$ requires $715.3271[\mathrm{M}+\mathrm{H}]^{+}$.

\section{4(R),5(R),6(R),7(R)-Tetra-benzyloxy-3,4,5,6,7,8,10,11,12-nonahydro-14-methoxy-1H-2,9-}

\section{benzdioxa-cyclotetradecin-1-one 9c}

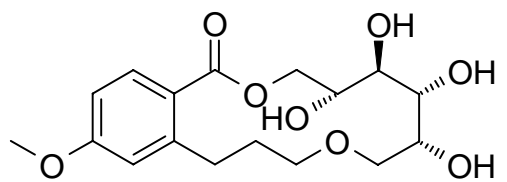

Catalytic hydrogenation of 24c (19 $\mathrm{mg}, 0.027 \mathrm{mmol})$ as described for 9a gave 9c $(4.5 \mathrm{mg}$, 47\%, oil) ; $\mathrm{R}_{\mathrm{f}} 0.18$ (EtOAc); ${ }^{1} \mathrm{H}$ NMR (500 MHz, MeOD): $\delta 7.88$ (d, $\left.1 \mathrm{H}, J 8.9 \mathrm{~Hz}\right), 6.86$ (d, 1H, $J 2.5 \mathrm{~Hz}), 6.83$ (dd, 1H, J $8.7 \& 2.6 \mathrm{~Hz}), 4.58$ (dd, 1H, J $11.5 \& 2.1 \mathrm{~Hz}), 4.49$ (dd, $1 \mathrm{H}, J$ 
$8.0 \& 3.7 \mathrm{~Hz}), 4.39$ (dd, 1H, J 11.5 \& $4.6 \mathrm{~Hz}), 4.13(\mathrm{~m}, 1 \mathrm{H}), 4.01$ (dd, 1H, J 3.6 \& $2.8 \mathrm{~Hz})$, 3.87 (ddd, 1H, J 7.6, 4.5 \& 2.1 Hz), 3.84 (s, 3H), 3.71 (dd, 1H, J 9.4 \& 7.1 Hz), 3.59 (m, 1H), $3.53(\mathrm{~m}, 2 \mathrm{H}), 3.23(\mathrm{~m}, 1 \mathrm{H}), 3.10(\mathrm{~m}, 1 \mathrm{H}), 1.87(\mathrm{~m}, 2 \mathrm{H}) ;{ }^{13} \mathrm{C} \mathrm{NMR}\left(\mathrm{CDCl}_{3}\right): \delta 170.4(\mathrm{C}=\mathrm{O})$, 164.3 (s), 146.7 (s), 134.9 (d), 123.6 (s), 116.6 (d), 112.5 (d), 73.9 (d), 73.1 (d), 72.8 (t), 72.0 (t), $71.7(\mathrm{~d}), 71.1(\mathrm{~d}), 67.2(\mathrm{t}), 55.9$ (q), 33.1 (t), 32.9 (t) ; IR (film, MeOH): $v_{\max }$ 3041, 2915, 2871, 1695, 1604, 1567, 1461, 1268, 1132, 1072, $1025 \mathrm{~cm}^{-1}$. ES-HRMS: Found 355.1387, $\mathrm{C}_{17} \mathrm{H}_{23} \mathrm{O}_{8}$ requires $355.1393[\mathrm{M}-\mathrm{H}]^{-}$.

\section{1-O-Allyl-6-O-(2-vinyl-benzoyl)-2,3,4,5-tetra-O-methoxymethyl-D-mannitol}

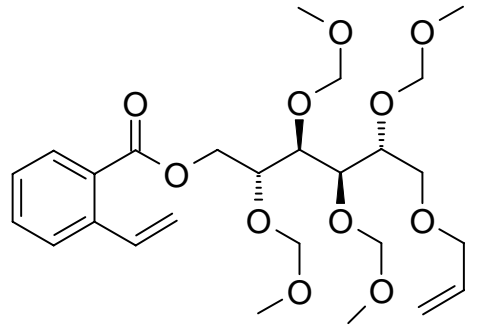

To reaction of 14a (105 $\mathrm{mg}, 0.26 \mathrm{mmol})$ and $22(43 \mathrm{mg}, 0.29 \mathrm{mmol})$ as described above for 14a and 19 gave the title compound ( $133 \mathrm{mg}, 97 \%$, colourless oil); $\mathrm{R}_{\mathrm{f}} 0.28$ (4:1 cyclohexaneEtOAc); $[\alpha]^{20}{ }_{\mathrm{D}}+33\left(\right.$ c $\left.1.0, \mathrm{CHCl}_{3}\right) ;{ }^{1} \mathrm{H} \mathrm{NMR}\left(600 \mathrm{MHz}, \mathrm{CDCl}_{3}\right): \delta 7.92(\mathrm{~d}, 1 \mathrm{H}, J 7.8 \mathrm{~Hz})$, $7.58(\mathrm{~d}, 1 \mathrm{H}, J 7.8 \mathrm{~Hz}), 7.51(\mathrm{dd}, 1 \mathrm{H}, J 17.5 \& 11.0 \mathrm{~Hz}), 7.48(\mathrm{t}, 1 \mathrm{H}, J 7.0 \mathrm{~Hz}), 7.31(\mathrm{t}, 1 \mathrm{H}, J$ $7.6 \mathrm{~Hz}), 5.90$ (ddd, 1H, J 22.8, $10.8 \& 5.6 \mathrm{~Hz}), 5.65$ (d, 1H, J $17.4 \mathrm{~Hz}), 5.35$ (d, 1H, J 11.0 Hz), $5.27(\mathrm{dd}, 1 \mathrm{H}, J 17.2 \& 1.5 \mathrm{~Hz}), 5.15(\mathrm{~d}, 1 \mathrm{H}, J 11.5 \mathrm{~Hz}), 4.78(\mathrm{~m}, 8 \mathrm{H}), 4.71(\mathrm{dd}, 1 \mathrm{H}, J$ $12.0 \& 2.8 \mathrm{~Hz}), 4.47(\mathrm{dd}, 1 \mathrm{H}, J 12.0 \& 5.9 \mathrm{~Hz}), 4.11(\mathrm{td}, 1 \mathrm{H}, J 5.4 \& 2.8 \mathrm{~Hz}), 4.07(\mathrm{t}, 1 \mathrm{H}, J$ $4.7 \mathrm{~Hz}), 4.00$ (m, 3H), 3.94 (dd, 1H, J $9.1 \& 4.9 \mathrm{~Hz}), 3.76$ (dd, 1H, J 10.4 \& $4.0 \mathrm{~Hz}), 3.62(\mathrm{dd}$, $1 \mathrm{H}, J 10.4 \& 5.1 \mathrm{~Hz}), 3.42(\mathrm{~s}, 3 \mathrm{H}), 3.41(\mathrm{~s}, 3 \mathrm{H}), 3.38(\mathrm{~s}, 3 \mathrm{H}), 3.35(\mathrm{~s}, 3 \mathrm{H}) ;{ }^{13} \mathrm{C} \mathrm{NMR}$ $\left(\mathrm{CDCl}_{3}\right): \delta 167.0(\mathrm{C}=\mathrm{O}), 139.5(\mathrm{~s}), 135.8(\mathrm{~d}), 134.5(\mathrm{~s}), 131.9,130.1$ (each d), $128.5(\mathrm{~s})$, $127.2(\mathrm{~d}), 127.0$ (d), $116.8(\mathrm{t}), 116.2$ (t), 97.9, 97.8, 96.4, 94.4 (each t), 77.2, 77.1, 76.8, 76.2 (each d), 72.1, 69.4, 64.7 (each t), 56.0, 55.9, 55.6, 55.5 (each q); IR (film, $\mathrm{CHCl}_{3}$ ): $v_{\max }$ 2925, 
2902, 2850, 1722, 1454, 1257, 1151, 1097, 1029, 919, 755, 665, $576 \mathrm{~cm}^{-1}$. ES-HRMS: Found 551.2464, $\mathrm{C}_{26} \mathrm{H}_{40} \mathrm{O}_{11} \mathrm{Na}$ requires $551.2468[\mathrm{M}+\mathrm{Na}]^{+}$.

\section{4(R),5(R),6(R),7(R)-Tetra-methoxymethoxy-3,4,5,6,7,8,10-heptahydro-[11E]-1H-2,9-}

\section{benzdioxa-cyclotetradecin-1-one 25}

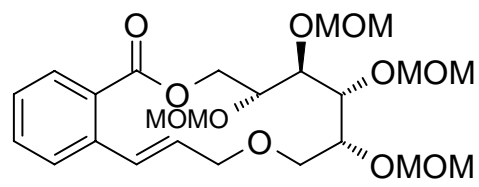

To a degassed solution of 1-O-allyl-6-O-(2-vinyl-benzoyl)-2,3,4,5-tetra-O-methoxymethyl-Dmannitol $(201 \mathrm{mg}, 0.38 \mathrm{mmol})$ in dry toluene $(119 \mathrm{~mL})$ was added $\mathrm{Ti}(\mathrm{OiPr})_{4}(34 \mu \mathrm{L}, 0.11$ mmol) at room temperature under $\mathrm{N}_{2}$. The mixture was stirred for $30 \mathrm{~min}$ and Grubbs'catalyst II generation (65 $\mathrm{mg}, 0.076 \mathrm{mmol})$ was added. The reaction mixture was gradually heated to $80{ }^{\circ} \mathrm{C}$ and stirred at that temperature for $14 \mathrm{~h}$. The solvent was removed under reduced pressure and the residue purified by chromatography $(1: 1$, cyclohexaneEtOAc) to give 25 (53 mg, 28\%, colourless oil, $\mathrm{R}_{\mathrm{f}} 0.31$ (3:7 cyclohexane-EtOAc)); ${ }^{1} \mathrm{H}$ NMR (400 MHz, MeOD): $\delta 7.93(\mathrm{~m}, 1 \mathrm{H}), 7.60-7.33(\mathrm{~m}, 4 \mathrm{H}), 6.12$ (d, 1H, J 13.9 Hz), 4.81-4.68 (m, 8H), $4.64(\mathrm{~m}, 2 \mathrm{H}), 4.55(\mathrm{~m}, 2 \mathrm{H}), 4.16(\mathrm{~m}, 1 \mathrm{H}), 4.10(\mathrm{dd}, 1 \mathrm{H}, J 6.3$ \& $1.3 \mathrm{~Hz}), 3.97(\mathrm{~m}, 1 \mathrm{H})$, $3.83(\mathrm{~m}, 1 \mathrm{H}), 3.64(\mathrm{dd}, 1 \mathrm{H}, J 10.7 \& 4.3 \mathrm{~Hz}), 3.44-3.21(\mathrm{~m}, 8 \mathrm{H}) ;{ }^{13} \mathrm{C} \mathrm{NMR}(\mathrm{MeOD}): \delta 170.1$ $(\mathrm{C}=\mathrm{O}), 139.6(\mathrm{~s}), 133.1$ (s), 132.4 (d), 131.9 (d), 128.3 (s \& d), 127.7 (d), 127.3 (d), 98.5 (t), $97.2(\mathrm{t}), 96.9(\mathrm{t}), 95.5(\mathrm{t}), 78.5(\mathrm{~d}), 77.2(\mathrm{~d}), 76.2(\mathrm{~d}), 69.6(\mathrm{t}), 68.9(\mathrm{t}), 55.6(\mathrm{q}), 55.1(\mathrm{q}), 54.7$ (q); IR (film, $\mathrm{CHCl}_{3}$ ): $v_{\max }$ 2946, 2929, 2890, 2360, 2341, 1708, 1598, 1477, 1448, 1369, 1290, 1259, 1209, 1151, 1101, 1027, 917, 792, 746, 665, $570 \mathrm{~cm}^{-1}$. ES-HRMS: Found 523.2136, $\mathrm{C}_{24} \mathrm{H}_{36} \mathrm{O}_{11} \mathrm{Na}$ requires $523.2155[\mathrm{M}+\mathrm{Na}]^{+}$. 
6-O-Allyl-2,3,4,5-tetra-O-benzyl-1-O-(2-vinylbenzoyl)-D-glucitol and 1-O-allyl-2,3,4,5tetra-O-benzyl-6-O-(2-vinylbenzoyl)-D-glucitol

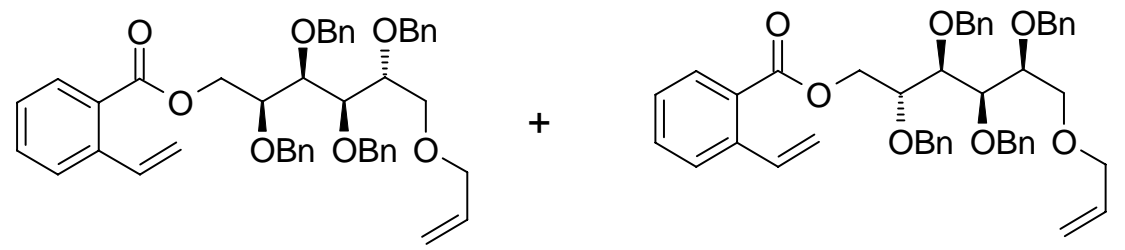

Reaction of the 1:1 mixture of 20 and 21 (722 mg, $1.24 \mathrm{mmol})$ and 14a (220 mg, $1.49 \mathrm{mmol}$, 1.2 eq.) as described for $\mathbf{1 9}$ and 14a gave an inseparable 3:2 mixture of the title compounds (671 mg, 76\%, colourless oil); $\mathrm{R}_{\mathrm{f}} 0.42$ (4:1 cyclohexane-EtOAc); ${ }^{1} \mathrm{H}$ NMR (500 MHz, $\left.\mathrm{CDCl}_{3}\right): \delta 7.79(\mathrm{dd}, 1 \mathrm{H}, J 7.8 \& 0.9 \mathrm{~Hz}), 7.75(\mathrm{dd}, 1 \mathrm{H}, J 7.8 \& 0.9 \mathrm{~Hz}), 7.57(\mathrm{~m}, 2 \mathrm{H}), 7.45$ (m, 4H), $7.27(\mathrm{~m}, 42 \mathrm{H}), 5.84(\mathrm{~m}, 2 \mathrm{H}), 5.62(\mathrm{~m}, 2 \mathrm{H}), 5.25(\mathrm{~m}, 4 \mathrm{H}), 5.12(\mathrm{~m}, 2 \mathrm{H}), 4.79(\mathrm{~m}$, $3 \mathrm{H}), 4.63(\mathrm{~m}, 14 \mathrm{H}), 4.48(\mathrm{dd}, 1 \mathrm{H}, J 12.1 \& 6.1 \mathrm{~Hz}), 4.43(\mathrm{~m}, 2 \mathrm{H}), 4.36(\mathrm{dd}, 1 \mathrm{H}, J 11.8$ \& 6.2 Hz), 4.06 (t, 1H, J 5.0 Hz), $4.02(\mathrm{t}, 1 \mathrm{H}, J 4.9 \mathrm{~Hz}), 3.96(\mathrm{~m}, 3 \mathrm{H}), 3.86(\mathrm{~m}, 7 \mathrm{H}), 3.66(\mathrm{~m}, 1 \mathrm{H})$, $3.61(\mathrm{dd}, 1 \mathrm{H}, J 10.4 \& 3.8 \mathrm{~Hz}), 3.52(\mathrm{dd}, 1 \mathrm{H}, J 10.4 \& 5.7 \mathrm{~Hz}) ;{ }^{13} \mathrm{C} \mathrm{NMR}\left(\mathrm{CDCl}_{3}\right): \delta 167.1$ $(\mathrm{C}=\mathrm{O}), 166.9(\mathrm{C}=\mathrm{O}), 139.7(\mathrm{~s}), 139.6(\mathrm{~s}), 138.6(\mathrm{~s}), 138.52(\mathrm{~s}), 138.49(\mathrm{~s}), 138.46(\mathrm{~s}), 138.43$ (s), 138.39 (s), 138.21 (s), 138.18 (s), 135.9 (d), [134.8, 134.7, 132.03, 131.99, 130.34, $130.32,128.7,128.5,128.3,128.25,128.23,128.21,128.18,128.17,128.03,128.00,127.91$, $127.88,127.58,127.56,127.54,127.53,127.50,127.48,127.35,127.29,127.12,127.09]$ (each d), 116.83 (d), 116.78 (d), 116.36 (d), 116.32 (d), [79.1, 79.0, 78.8, 78.7, 78.6, 78.5, 78.3, 77.6] (each d), $[74.8,74.7,74.3,73.9,73.0,72.9,72.2,72.1,72.0,71.8,70.2,69.5,64.5$, 64.2] (each t) ; IR (film, $\mathrm{CHCl}_{3}$ ) : $v_{\max }$ 3064, 3029, 2919, 2873, 2360, 2341, 1720, 1600, 1496, 1454, 1255, 1205, 1089, 1066, 916, 757, 736, $701 \mathrm{~cm}^{-1}$. ES-HRMS: Found 735.3311, $\mathrm{C}_{46} \mathrm{H}_{48} \mathrm{O}_{7} \mathrm{Na}$ requires $735.3298[\mathrm{M}+\mathrm{Na}]^{+}$. 


\section{4(S),5(R),6(R),7(R)-Tetra-benzyloxy-3,4,5,6,7,8,10-heptahydro-[11E]-1H-2,9-benzdioxa-}

cyclotetradecin-1-one 26 and $4(R), 5(R), 6(R), 7(S)$-tetra-benzyloxy-3,4,5,6,7,8,10heptahydro-[11E]-1H-2,9-benzdioxa-cyclotetradecin-1-one 27

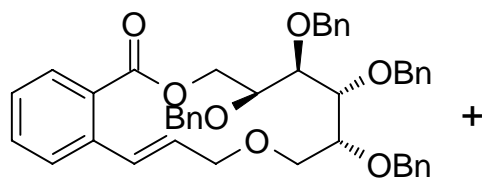

26

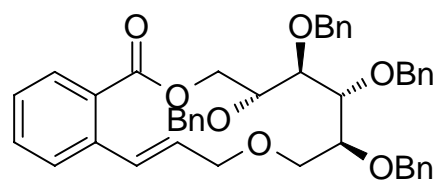

27

To a degassed mixture of 6-O-allyl-2,3,4,5-tetra-O-benzyl-1-O-(2-vinylbenzoyl)-D-glucitol and 1-O-allyl-2,3,4,5-tetra-O-benzyl-6-O-(2-vinylbenzoyl)-D-glucitol $(631 \mathrm{mg}, 0.89 \mathrm{mmol})$ and 2,6-dichloro-1,4-benzoquinone $(16 \mathrm{mg}, 0.089 \mathrm{mmol})$ in dry toluene $(445 \mathrm{~mL})$ was added Grubbs' catalyst II generation $\left(38 \mathrm{mg}, 0.044 \mathrm{mmol}\right.$ ) under $\mathrm{N}_{2}$ atmosphere at room temperature. The reaction mixture was gradually heated to $70{ }^{\circ} \mathrm{C}$ and left stirring at that temperature for $17 \mathrm{~h}$. The solution was then filtered through a pad of silica, washed with toluene and concentrated in vacuo. The residue was purify by chromatography $(9: 1$, cyclohexane-EtOAc) to give $26\left(173 \mathrm{mg}, 40 \%\right.$, colourless oil, $\mathrm{R}_{\mathrm{f}} 0.16$ (9:1 cyclohexaneEtOAc)) and 27 (191 mg, 44\%, colourless, $\mathrm{R}_{\mathrm{f}} 0.13$ (9:1 cyclohexane-EtOAc)). Analytical data for the 26: $[\alpha]^{20}{ }_{\mathrm{D}}+3\left(c 0.53, \mathrm{CHCl}_{3}\right) ;{ }^{1} \mathrm{H}$ NMR $\left(500 \mathrm{MHz}, \mathrm{CDCl}_{3}\right): \delta 7.89(\mathrm{~d}, 1 \mathrm{H}, J 8.1$ Hz), $7.33(\mathrm{~m}, 21 \mathrm{H}), 7.05(\mathrm{~m}, 2 \mathrm{H}), 6.96(\mathrm{t}, 1 \mathrm{H}, J 7.4 \mathrm{~Hz}), 6.07(\mathrm{dt}, 1 \mathrm{H}, J 16.0 \& 3.5 \mathrm{~Hz}), 4.97$ $(\mathrm{d}, 1 \mathrm{H}, J 11.1 \mathrm{~Hz}), 4.88(\mathrm{~d}, 1 \mathrm{H}, J 11.4 \mathrm{~Hz}), 4.84(\mathrm{~d}, 1 \mathrm{H}, J 11.1 \mathrm{~Hz}), 4.71(\mathrm{~d}, 1 \mathrm{H}, J 11.9 \mathrm{~Hz})$, $4.65(\mathrm{dd}, 1 \mathrm{H}, J 10.4 \& 3.3 \mathrm{~Hz}), 4.59$ (d, 1H, J 11.4 Hz), 4.47 (m, 1H), 4.44 (d, 1H, J $11.9 \mathrm{~Hz}$, $\left.\mathrm{CH}_{2} \mathrm{Ph}\right), 4.43(\mathrm{~d}, 1 \mathrm{H}, J 12.1 \mathrm{~Hz}), 4.36(\mathrm{~d}, 1 \mathrm{H}, J 12.1 \mathrm{~Hz}), 4.24(\mathrm{t}, 1 \mathrm{H}, J 10.3 \mathrm{~Hz}), 4.21(\mathrm{dd}$, $1 \mathrm{H}, J 9.1 \& 1.6 \mathrm{~Hz}), 4.17(\mathrm{dd}, 2 \mathrm{H}, J 5.7 \& 3.5 \mathrm{~Hz}), 4.06(\mathrm{t}, 1 \mathrm{H}, J 9.2 \mathrm{~Hz}), 3.92(\mathrm{ddd}, 1 \mathrm{H}, J$ $10.2,3.2 \& 1.8 \mathrm{~Hz}), 3.77(\mathrm{dd}, 1 \mathrm{H}, J 8.8 \& 3.5 \mathrm{~Hz}), 3.67(\mathrm{dd}, 1 \mathrm{H}, J 9.5 \& 3.5 \mathrm{~Hz}) ;{ }^{13} \mathrm{C} \mathrm{NMR}$ $\left(\mathrm{CDCl}_{3}\right): \delta 167.4(\mathrm{C}=\mathrm{O}), 139.4(\mathrm{~s}),[138.5,138.2,137.78,137.75]$ (each s), $132.2(\mathrm{~d}), 131.4$ (d), 129.4 (s), [128.8, 128.6, 128.5, 128.3, 128.2, 128.1, 128.0, 127.9, 127.5, 127.4, 127.3, $127.1,127.0,126.9,126.4,82.3,77.8,77.5,76.6]$ (each d), $[75.6,75.5,72.3,71.5,70.0,68.1$, 
60.3] (each t) ; IR (film, $\mathrm{CHCl}_{3}$ ): $v_{\max }$ 3087, 3062, 3029, 2913, 2869, 2360, 2341, 2246, 1951, $1710,1598,1496,1454,1288,1259,1207,1132,971,908,854,809,744,698,572 \mathrm{~cm}^{-1} . \mathrm{ES}-$ HRMS: Found 707.2985, $\mathrm{C}_{44} \mathrm{H}_{44} \mathrm{O}_{7} \mathrm{Na}$ requires $707.2985[\mathrm{M}+\mathrm{Na}]^{+}$. Analytical data for 27: $\left[\alpha{ }^{20}{ }_{\mathrm{D}}-13\left(\mathrm{c} 0.56, \mathrm{CHCl}_{3}\right) ;{ }^{1} \mathrm{H}\right.$ NMR $\left(500 \mathrm{MHz}, \mathrm{CDCl}_{3}\right): \delta 7.88$ (dd, $\left.1 \mathrm{H}, J 7.8 \& 0.9 \mathrm{~Hz}\right)$, $7.49(\mathrm{dd}, 1 \mathrm{H}, J 7.8 \& 1.2 \mathrm{~Hz}), 7.46(\mathrm{ddd}, 1 \mathrm{H}, J$ 8.0, 7.6 \& $1.0 \mathrm{~Hz}), 7.26\left(\mathrm{~m}, 22 \mathrm{H}, \mathrm{H}_{\text {benzyl }}\right), 6.11$ $(\mathrm{dt}, 1 \mathrm{H}, J 15.9 \& 4.9 \mathrm{~Hz}), 5.17(\mathrm{dd}, 1 \mathrm{H}, J 12.2 \& 2.3 \mathrm{~Hz}), 4.98(\mathrm{~d}, 1 \mathrm{H}, J 11.8 \mathrm{~Hz}), 4.74(\mathrm{~d}$, 1H, J $11.8 \mathrm{~Hz}), 4.67(\mathrm{~m}, 4 \mathrm{H}), 4.61(\mathrm{~d}, 1 \mathrm{H}, J 11.8 \mathrm{~Hz}), 4.49$ (dd, 1H, J $8.6 \& 1.4 \mathrm{~Hz}), 4.28(\mathrm{~d}$, $1 \mathrm{H}, J 12.1 \mathrm{~Hz}), 4.21(\mathrm{~d}, 1 \mathrm{H}, J 8.9 \mathrm{~Hz}), 4.15(\mathrm{~m}, 2 \mathrm{H}), 4.07$ (ddd, 1H, J 13.7, 4.6 \& $1.2 \mathrm{~Hz})$, 3.89 (ddd, 1H, J 9.2, $6.2 \& 3.4 \mathrm{~Hz}$ ), 3.85 (dd, 1H, J $8.6 \& 1.4 \mathrm{~Hz}), 3.69$ (dd, 1H, J 10.6 \& 3.4 $\mathrm{Hz}), 3.48(\mathrm{dd}, 1 \mathrm{H}, J 10.6 \& 6.3 \mathrm{~Hz}) ;{ }^{13} \mathrm{C} \mathrm{NMR}\left(\mathrm{CDCl}_{3}\right): \delta 168.4(\mathrm{C}=\mathrm{O}),[139.4,138.5,138.4$, 138.0, 137.4] (each s), 132.2 (d), 131.9 (d), 131.3 (d), 129.8 (s), [128.3, 128.20, 128.17, $128.0,127.92,127.90,127.7,127.6,127.5,127.38,127.35,127.2,127.1,80.0,78.5,76.8$, 75.9] (each d), [75.2, 73.3, 71.2, 70.8, 70.5, 61.1] (each t) ; IR (film, $\mathrm{CHCl}_{3}$ ): $v_{\max } 3087,3062$, $3029,2915,2865,2362,2337,1955,1712,1598,1496,1454,1290,1259,1207,1124,1089$, 1027, 971, 910, 840, 734, 696, $646 \mathrm{~cm}^{-1}$. ES-HRMS: Found 707.3004, $\mathrm{C}_{44} \mathrm{H}_{44} \mathrm{O}_{7} \mathrm{Na}$ requires $707.2985[\mathrm{M}+\mathrm{Na}]^{+}$.

\section{4(S),5(R),6(R),7(R)-Tetrahydroxy-3,4,5,6,7,8,10,11,12-nonahydro-1H-2,9-benzdioxa-} cyclotetradecin-1-one 11

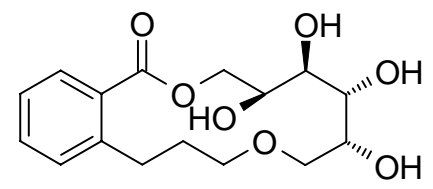

Catalytic hydrogenation of $26(96 \mathrm{mg}, 0.14 \mathrm{mmol})$ in $\mathrm{EtOH}(5.6 \mathrm{~mL})$ as described above gave 11 (20 mg, 48\%); $\mathrm{R}_{\mathrm{f}} 0.18\left(9: 1, \mathrm{CH}_{2} \mathrm{Cl}_{2}-\mathrm{MeOH}\right) ;{ }^{1} \mathrm{H}$ NMR (600 MHz, DMSO): $\delta 7.74$ $(\mathrm{dd}, 1 \mathrm{H}, J 7.8 \& 1.2 \mathrm{~Hz}), 7.48(\mathrm{td}, 1 \mathrm{H}, J 7.6 \& 1.3 \mathrm{~Hz}), 7.35(\mathrm{dd}, 1 \mathrm{H}, J 7.9 \& 0.8 \mathrm{~Hz}), 7.30$ (td, 1H, J $7.7 \& 1.0 \mathrm{~Hz}), 4.69(\mathrm{~d}, 1 \mathrm{H}, J 7.2 \mathrm{~Hz}), 4.55(\mathrm{~d}, 1 \mathrm{H}, J 6.5 \mathrm{~Hz}), 4.42(\mathrm{~d}, 1 \mathrm{H}, J 4.6 \mathrm{~Hz})$, 
4.37 (d, 1H, J $5.8 \mathrm{~Hz}), 4.28$ (t, 1H, J $9.9 \mathrm{~Hz}), 4.21$ (dd, 1H, J 10.3 \& $3.4 \mathrm{~Hz}), 4.03$ (m, 1H), $3.83(\mathrm{~m}, 2 \mathrm{H}), 3.77(\mathrm{~m}, 1 \mathrm{H}), 3.56(\mathrm{dd}, 1 \mathrm{H}, J 9.5 \& 8.5 \mathrm{~Hz}), 3.47$ (ddd, 1H, J 8.6, $5.7 \& 2.5$ Hz), 3.27 (m, 1H), 3.22 (ddd, 1H, J 9.0, $8.9 \& 1.7 \mathrm{~Hz}), 3.14$ (td, 1H, J $12.3 \& 4.2 \mathrm{~Hz}), 2.75$ $(\mathrm{td}, 1 \mathrm{H}, J 12.3 \& 5.7 \mathrm{~Hz}), 1.70(\mathrm{~m}, 1 \mathrm{H}), 1.60(\mathrm{~m}, 1 \mathrm{H}) ;{ }^{13} \mathrm{C}$ NMR (DMSO): $\delta 167.9(\mathrm{C}=\mathrm{O})$, 142.0 (s), 131.7 (d), 130.8 (d), 130.4 (d), 130.0 (s), 125.8 (d), 74.1 (d), 70.5 (d), 70.1 (t), 69.5 (t), $68.5(\mathrm{~d}), 67.1(\mathrm{~d}), 64.8(\mathrm{t}), 33.0(\mathrm{t}), 30.9$ (t); IR (film, DMSO) : $v_{\max }$ 2967, 2356, 2339, $1635,1614,1261,1089,1022,802 \mathrm{~cm}^{-1}$. ES-HRMS: Found 349.1279, $\mathrm{C}_{16} \mathrm{H}_{22} \mathrm{O}_{7} \mathrm{Na}$ requires $349.1263[\mathrm{M}+\mathrm{Na}]^{+}$.

\section{4(R),5(R),6(R),7(S)-Tetrahydroxy-3,4,5,6,7,8,10,11,12-nonahydro-1H-2,9-benzdioxa-}

cyclotetradecin-1-one 12

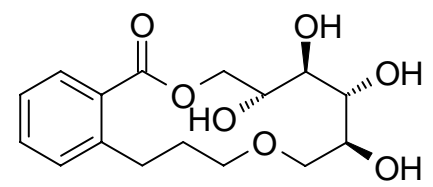

Catalytic hydrogenation of $27(138 \mathrm{mg}, 0.20 \mathrm{mmol})$ in EtOH $(8 \mathrm{~mL})$ as described above gave 12 initially as colourless oil (58 mg, 89\%); $\mathrm{R}_{\mathrm{f}} 0.33\left(9: 1, \mathrm{CH}_{2} \mathrm{Cl}_{2}-\mathrm{MeOH}\right)$, and ultimately as crystals : mp 136.5-140 ${ }^{\circ} \mathrm{C}(\mathrm{MeOH}) ;{ }^{1} \mathrm{H}$ NMR (500 MHz, MeOD): $\delta 7.76(\mathrm{dd}, 1 \mathrm{H}, J 7.8 \&$ $1.2 \mathrm{~Hz}), 7.49$ (td, $1 \mathrm{H}, J 7.7 \& 1.4 \mathrm{~Hz}), 7.40$ (d, 1H, J $7.7 \mathrm{~Hz}), 7.29(\mathrm{td}, 1 \mathrm{H}, J 7.8$ \& $1.0 \mathrm{~Hz})$, $4.71(\mathrm{dd}, 1 \mathrm{H}, J 11.5 \& 2.4 \mathrm{~Hz}), 4.49(\mathrm{dd}, 1 \mathrm{H}, J 11.5 \& 4.6 \mathrm{~Hz}), 4.18(\mathrm{dd}, 1 \mathrm{H}, J 7.8$ \& $1.2 \mathrm{~Hz})$ 4.00 (m, 1H), $3.98(\mathrm{dd}, 1 \mathrm{H}, J 8.6 \& 1.2 \mathrm{~Hz}), 3.83(\mathrm{ddd}, 1 \mathrm{H}, J$ 8.6, $6.1 \& 2.6 \mathrm{~Hz}), 3.72(\mathrm{dd}, 1 \mathrm{H}$, $J 9.6 \& 2.6 \mathrm{~Hz}), 3.56(\mathrm{~m}, 2 \mathrm{H}), 3.46(\mathrm{dd}, 1 \mathrm{H}, J 9.6 \& 6.2 \mathrm{~Hz}), 3.20(\mathrm{~m}, 1 \mathrm{H}), 3.07(\mathrm{~m}, 1 \mathrm{H}), 1.92$ $(\mathrm{m}, 2 \mathrm{H}) ;{ }^{13} \mathrm{C}$ NMR (MeOD): $\delta 171.0(\mathrm{C}=\mathrm{O}), 143.2$ (s), 132.9 (d), 132.5 (s), 131.5 (d), 131.0 (d), $126.9(\mathrm{~d}), 74.1(\mathrm{t}), 73.9(\mathrm{~d}), 72.4(\mathrm{~d}), 71.7(\mathrm{t}), 71.2(\mathrm{~d}), 69.7(\mathrm{~d}), 67.3(\mathrm{t}), 32.1(\mathrm{t}), 31.9(\mathrm{t})$; IR (film, MeOD) : $v_{\max } 3382,2929,2871,2256,2127,1704,1600,1529,1446,1371,1286$, 1263, 1178, 1132, 1089, 1049, 1025, 1000, 825, $761 \mathrm{~cm}^{-1}$. ES-HRMS: Found 349.1280, $\mathrm{C}_{16} \mathrm{H}_{22} \mathrm{O}_{7} \mathrm{Na}$ requires $349.1263[\mathrm{M}+\mathrm{Na}]^{+}$. 


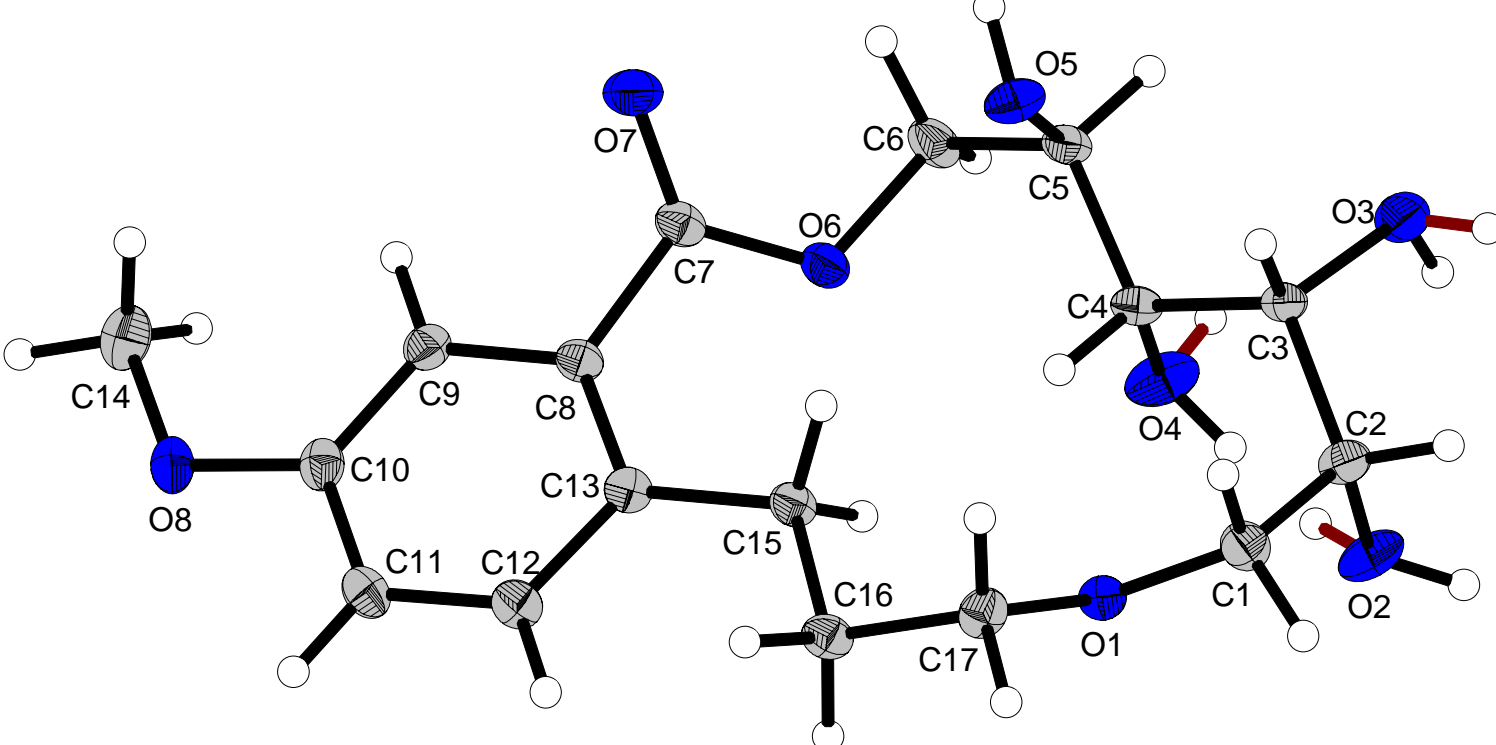

Figure 1. X-ray crystal structure of $\mathbf{9 b}$. Thermal ellipsoids are drawn on the $50 \%$ probability level

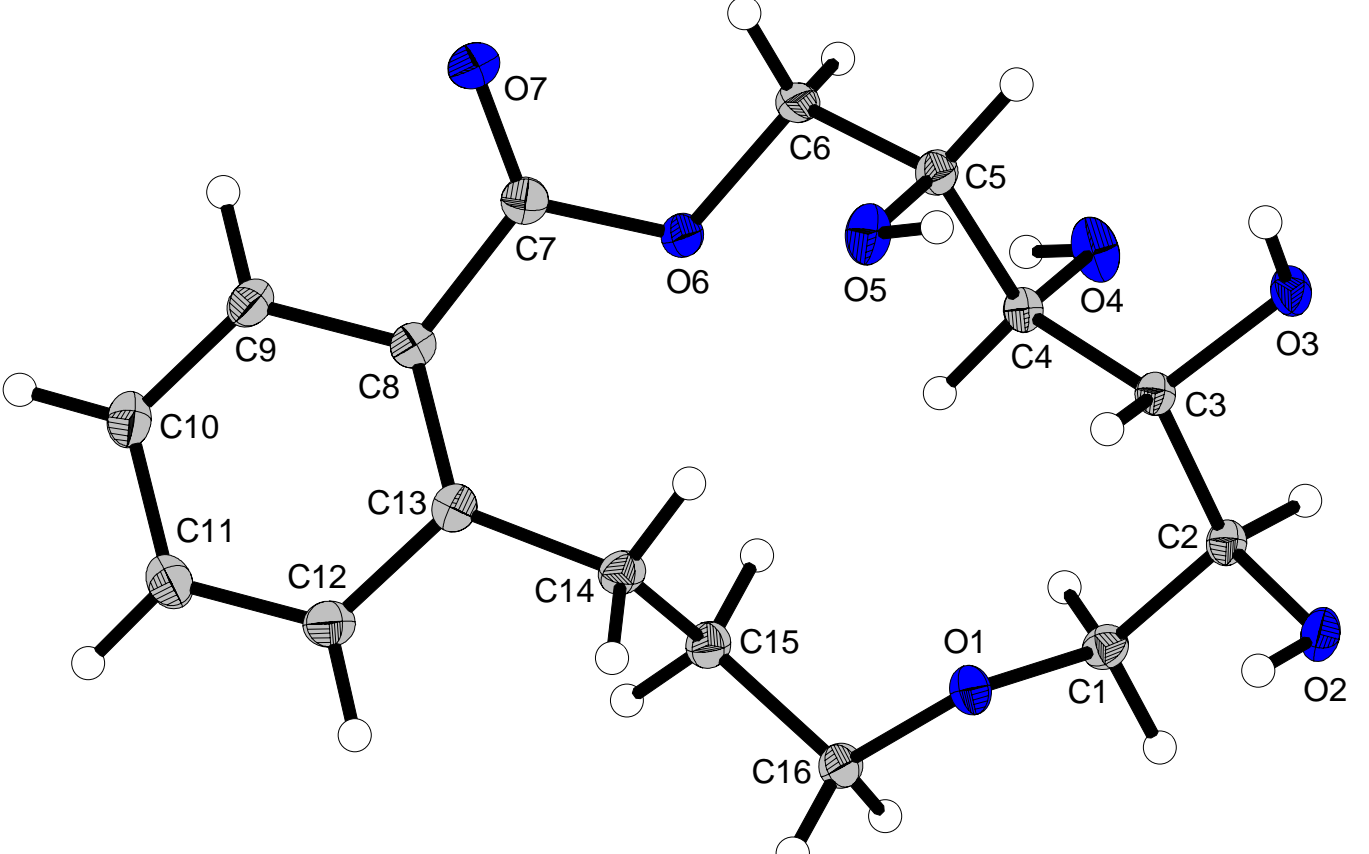

Figure 2. X-ray crystal structure of 12. Thermal ellipsoids are drawn on the $50 \%$ probability level. 